\title{
The Almost Rigidity of Manifolds with Lower Bounds on Ricci Curvature and Minimal Volume Growth
}

Christina SoRMANI ${ }^{1}$

Twenty years ago, Calabi and Yau each proved that a complete noncompact Riemannian manifold with nonnegative Ricci curvature must have at least linear volume growth [Yau]. This was proven by studying the Busemann function, $b=b_{\gamma}$, associated with a ray, $\gamma$,

$$
b(x)=\lim _{R \rightarrow \infty}(R-d(x, \gamma(R))
$$

In [So2], the author proved that if such a manifold has linear volume growth then its Busemann functions are proper. The simplest examples of manifolds with linear volume growth are the metric product manifolds, $X \times \mathbb{R}$, whose cross sections, $X \times\{r\}$, are level sets of the Busemann functions.

In this paper we prove that a complete noncompact manifold with nonnegative Ricci curvature and linear volume growth must be close to being such a metric product manifold asymptotically [Theorem 34]. That is, as $r \rightarrow \infty$, the set $b^{-1}([r, r+L])$ becomes close to $b^{-1}(r) \times[r, r+L]$ in the Gromov-Hausdorff topology where the closeness depends linearly on $\operatorname{diam}\left(b^{-1}(r)\right)$. See Section 2. The proof involves a careful analysis of the Busemann function using the recently-developed Cheeger-Colding Almost Rigidity Theory [ChCo]. We also use this method to prove the following theorem.

Theorem 1. If $M^{n}$ is a manifold with nonnegative Ricci curvature and linear volume growth, then it has sublinear diameter growth,

$$
\lim _{R \rightarrow \infty} \frac{\operatorname{diam}\left(b^{-1}(R)\right)}{R}=0 .
$$

\footnotetext{
${ }^{1}$ This material is based upon work supported under a National Science Foundation Graduate Fellowship.
} 
Examples of manifolds satisfying the hypothesis of this theorem for which $\operatorname{diam}\left(b^{-1}(R)\right)$ grows logarithmicly appeared in [So2]. Applications of this theorem to the analysis of harmonic functions on manifolds with nonnegative Ricci curvature and linear volume growth will appear in [So3].

In addition to studying manifolds with nonnegative Ricci curvature, we study manifolds with a quadratically decaying lower bound on Ricci curvature,

$$
\operatorname{Ric}(x) \geq \frac{(n-1)\left(\frac{1}{4}-v^{2}\right)}{b(x)^{2}} \text { for all } x \in b^{-1}\left(\left[r_{0}, \infty\right)\right),
$$

where $v$ can take any value in $\left[0, \frac{n+1}{2(n-1)}\right)$. This bound is implied by the traditional quadratically decaying lower bound on Ricci curvature defined using the distance function from a base point [ChGrTa].

Such manifolds also have a lower bound on their volume growth,

$$
\liminf _{R \rightarrow \infty} \frac{\operatorname{Vol}\left(B_{x_{0}}(R)\right)}{R^{p}} \geq C>0,
$$

where $p=(n-1)(1 / 2-v)+1>0$ [ChGrTa]. If one is given the (n-1) Hausdorff volume of a compact set, $S \subset b^{-1}\left(r_{1}\right) \subset b^{-1}\left(\left(r_{0}, \infty\right)\right)$, then there is a precise lower bound, $C=C_{S}$, depending upon that volume [So2, see Corollary 7]. In section 1.1, we review this and a relative volume comparison theorem for Busemann functions that is needed for this paper.

Definition 2. We say that a manifold, $M^{n}$, has minimal volume growth if it has a quadratically decaying lower Ricci curvature bound as in (0.1) and

$$
\limsup _{R \rightarrow \infty} \frac{\operatorname{Vol}\left(B_{x_{0}}(R)\right)}{R^{p}}=V_{0}<\infty
$$

where $p=\left(\frac{1}{2}-v\right)(n-1)+1$.

Note that a manifold with nonnegative Ricci curvature, $v=1 / 2$, has minimal volume growth if it has linear volume growth.

In contrast, we will say that a manifold satisfying (0.1) has strongly minimal volume growth with respect to the compact set, $S \subset b^{-1}\left(r_{1}\right)$, if

$$
\limsup _{R \rightarrow \infty} \frac{\left.\operatorname{Vol}\left(B_{x_{0}}(R)\right)\right)}{R^{p}}=C_{S}
$$

where $p=\left(\frac{1}{2}-v\right)(n-1)+1$ [see also Defn 8]. 
In Section 1.2 we state and prove a rigidity theorem for the end of a manifold with strongly minimal volume growth [Theorem 9]. First we show that the given level set, $b^{-1}\left(r_{1}\right)$, is a compact smoothly embedded submanifold. Then we prove the end of the manifold is a warped product,

$$
b^{-1}\left(\left[r_{1}, \infty\right)\right)=b^{-1}\left(r_{1}\right) \times_{\left(b / r_{1}\right)^{(1 / 2-v)}}\left[r_{1}, \infty\right) .
$$

That is, it has a metric of the form

$$
d b^{2}+\left(\frac{b}{r_{1}}\right)^{\left(\frac{1}{2}-v\right) 2} g_{0}
$$

where $g_{0}$ is the induced metric on $b^{-1}\left(r_{1}\right)$.

In particular, a manifold with Ricci $\geq 0, v=1 / 2$ and strongly minimal volume growth has only one end and that end is an isometric product [Cor 10].

In [So2], there are examples which demonstrate that the strongly minimal volume growth condition in Theorem 9 is necessary. These examples have nonnegative Ricci curvature and linear volume growth,

$$
\lim _{r \rightarrow \infty} \frac{\operatorname{Vol}\left(B_{x_{0}}(r)\right)}{r}=V_{0}<\infty,
$$

but their ends are not isometric product manifolds.

In the second section of this paper, we prove the corresponding almost rigidity theorem, Theorem 33. Here we add the assumption that Ricci $\geq 0$ everywhere on the manifold along with the quadratically decaying lower bound on Ricci curvature, (0.1), with $v$ now in $[0,1 / 2]$ and minimal volume growth. These conditions imply that the Busemann function is proper [So2, Theorem 19].

Theorem 33 roughly states that such a manifold is asymptotically close in the Gromov-Hausdorff sense to a warped product manifold with a metric as in (0.4). More precisely, we show that for $r$ sufficiently large, the compact region $b^{-1}([r, r+L])$ is close to a warped product, $X_{r} \times_{f}[r, r+L]$ with the warping function, $f(s)=(s / r)^{(1 / 2-v)}$, where $X_{r}$ is a length space close to $b^{-1}(r)$, [Theorem 33]. This closeness depends linearly on the diameter $b^{-1}(r)$.

It is important to note that a manifold with minimal volume growth and with globally nonnegative and quadratically decaying Ricci curvature is not asymptotically close to a unique warped product manifold. In particular a manifold with nonnegative Ricci curvature and linear volume growth has regions, $b^{-1}((r, r+L))$, which approach isometric product manifolds, $X_{r} \times$ 
$(r, r+L)$, but $X_{r}$ may change slowly as $r$ approaches infinity. Examples where $X_{r}$ alternates between two different Riemannian manifolds appear in [So2].

In order to precisely state Theorem 33 , we must define localized distance, the metric which we will be using on the regions, $b^{-1}([r, r+L])$. This definition and a definition of the Gromov-Hausdorff distance appear in Section 2.1. The statement of Theorem 33 and the related Theorem 34, along with a discussion of their consequences appears in Section 2.2. The proof appears in Section 2.4 after a Section 2.3, which relates Cheeger and Colding's work on almost rigidity and maximal volume to our condition of minimal volume growth.

Lastly, in Section 3 we focus on manifolds with globally nonnegative Ricci curvature, $v=1 / 2$, and linear volume growth. The same lemmas used to prove our almost rigidity theorem in Sections 2.1 and 2.3 are directly applied to prove two results on the diameter growth of such manifolds. In Theorem 45, we prove that the localized diameter of the level sets of the Busemann function grows sublinearly. This theorem is stated in Section 3 after defining localized diameter in Definition 44. This theorem is then employed to prove the Theorem 1 stated above, in which the diameter of the level sets are measured in the ambient manifold, $M^{n}$.

The author would like to thank Professor Cheeger for suggesting an analysis of manifolds with minimal volume growth and for numerous enlightening conversations. She would also like to thank Professor Colding for helpful discussions on his work in almost rigidity theory. Finally, she is very grateful to the Courant Institute of Mathematical Sciences for its generous support in her years as a graduate student.

Background material can be found in [ChEb], [BiCr] and [Ch].

\section{Strongly Minimal Volume Growth and Rigidity.}

\subsection{Background on Volume Growth.}

We now review some definitions and theorems regarding special sets in noncompact manifolds with quadratically decaying lower Ricci curvature bounds. See [So2]. This background will be used to study both the rigidity and almost rigidity of such manifolds later in this paper.

All geodesics and rays are parametized by arclength.

Definition 3. Let $\gamma$ be a ray, $b_{\gamma}(x)$ be its associated Busemann function and $x \in M^{n}$. A ray, $\gamma_{x}:[b(x), \infty) \mapsto M$, emanating from $x$ is called a 
Busemann ray associated with $\gamma$ if it is the limit of a sequence of minimal geodesics $\sigma_{i}$ from $x$ to $\gamma\left(R_{i}\right)$ in the following sense,

$$
\gamma_{x}^{\prime}(b(x))=\lim _{R_{i} \rightarrow \infty} \sigma_{i}^{\prime}(0)
$$

Note that $\gamma_{x}(b(x))=x$. Note also that $\gamma_{x}$ need not be unique.

Definition 4. Given a compact set, $K$, contained in $M^{n}$ and a ray, $\gamma$, let

$$
\Omega(K)=\left\{x: \exists z \in K \exists t \geq b(z) \text { s.t. } x=\gamma_{z}(t)\right\}
$$

Let

$$
\Omega_{s_{1}, s_{2}}(K)=\Omega(K) \cap b^{-1}\left(\left[s_{1}, s_{2}\right]\right) .
$$

In [So2, Cor 15], the author proved the following volume comparison theorem.

Theorem 5 (So2, Thm 5, Cor 17). Let

$$
R i c \geq(n-1)\left(\frac{1}{4}-v^{2}\right) / b(x)^{2}
$$

whenever $x \in b^{-1}\left(\left[r_{0}, \infty\right)\right)$ where $v \in\left[0, \frac{n+1}{2(n-1)}\right)$. Let $r_{1} \geq r_{0}$ and let $K$ be a compact set contained in $b^{-1}\left(\left(-\infty, r_{1}\right]\right)$.

Then there exists a nondecreasing function, $V(r)$, such that

$$
V(r)=\frac{V o l_{n-1}\left(\Omega(K) \cap b^{-1}(r)\right)}{r^{\left(\frac{1}{2}-v\right)(n-1)}}
$$

almost everywhere in $\left[r_{1}, \infty\right)$.

In particular, for almost every $s_{2}>s_{1} \geq r_{1}$ we have

$$
\frac{V\left(s_{1}\right)}{p} \leq \frac{V o l\left(\Omega(K) \cap b^{-1}\left(s_{1}, s_{2}\right)\right)}{s_{2}^{p}-s_{1}^{p}} \leq \frac{\left.V\left(s_{2}\right)\right)}{p}
$$

where $p=(1 / 2-v)(n-1)+1$.

This theorem was proven using the following series of comparison manifolds which were also employed in [ChGrTa]. 
Definition 6. The comparison manifold, $M_{R, \varepsilon}^{n}$, is a warped product manifold diffeomorphic to $\mathbb{R}^{n}$, with the metric $d t^{2}+J_{R, \varepsilon}(t)^{2} g_{0}$ where

$$
J_{R, \varepsilon}(t)=\frac{R+\varepsilon}{2 v}\left(-\left(\frac{R-t+\varepsilon}{R+\varepsilon}\right)^{\frac{1}{2}+v}+\left(\frac{R-t+\varepsilon}{R+\varepsilon}\right)^{\frac{1}{2}-v}\right)
$$

and $g_{0}$ is the standard metric on the sphere. Here $\varepsilon:=0$ when $v \geq 1 / 2$.

These manifolds satisfy the following Ricci curvature bound,

$$
R i c_{y} \geq \frac{(n-1)\left(\frac{1}{4}-v^{2}\right)}{(R-d(y, 0)+\varepsilon)^{2}}
$$

Note also that

$$
\lim _{R \rightarrow \infty} \frac{\int_{R-r_{4}}^{R-r_{3}} J_{R . \varepsilon}(t)^{n-1} d t}{\int_{R-r_{3}}^{R-r_{1}} J_{R . \varepsilon}(t)^{n-1} d t}=\frac{\left(r_{4}\right)^{p}-\left(r_{3}\right)^{p}}{\left(r_{3}\right)^{p}-\left(r_{1}\right)^{p}}
$$

These comparison manifolds, (1.5), and the Relative Volume Comparison Theorem $[\mathrm{BiCr}, \mathrm{GrLaPa}]$ will be used to prove our rigidity theorem.

The following corollary of Theorem 5, defines the precise constant, $C_{S}$, mentioned in the introduction and allows us to define strongly minimal volume growth for ends.

Corollary 7. Given $M^{n}$ as described in (0.1) and given any $R>r_{1} \geq r_{0}$ and any $R_{0}>0$, let $S=B_{x_{0}}\left(R_{0}\right) \cap b^{-1}\left(r_{1}\right)$. Then

$$
\operatorname{Vol}\left(B_{x_{0}}\left(R+R_{0}-r_{0}\right)\right) \geq C_{S}\left(R^{p}-r_{0}^{p}\right)
$$

where $p=\left(\frac{1}{2}-v\right)(n-1)+1>0$ and $C_{S}=\operatorname{Vol}_{n-1}(S) /\left(p r_{1}^{p-1}\right)$.

Definition 8. Given $r_{1} \geq r_{0}$, we say that a region, $b^{-1}\left(\left(r_{1}, \infty\right)\right)$, in a manifold, $M^{n}$, has strongly minimal volume growth with respect to a given ball $B_{x_{0}}\left(R_{0}\right)$, if it has a quadratically decaying lower Ricci curvature bound as in $(0.1)$ and

$$
\lim _{R \rightarrow \infty} \frac{\operatorname{Vol}\left(B_{x_{0}}(R) \cap b^{-1}\left(\left(r_{1}, \infty\right)\right)\right)}{R^{p}}=C_{S}
$$

where $S=B_{x_{0}}\left(R_{0}\right) \cap b^{-1}\left(r_{1}\right)$ and $p=\left(\frac{1}{2}-v\right)(n-1)+1$. 


\subsection{Strongly Minimal Volume Growth and Rigidity.}

In this section we prove the following rigidity theorem.

Theorem 9. Let $M^{n}$ be a complete noncompact manifold with a ray $\gamma$ and a Busemann function $b: M \rightarrow \mathbb{R}$ and a quadratically decaying lower Ricci curvature bound as in (0.1). Suppose $b^{-1}\left(\left(r_{1}, \infty\right)\right)$ has strongly minimal volume growth with respect to a given ball, $B_{x_{0}}\left(R_{0}\right)$. Then $b^{-1}\left(r_{1}\right)$ is a smoothly embedded submanifold contained in $\bar{B}_{R_{0}}(\gamma(0))$ and

$$
b^{-1}\left(\left[r_{1}, \infty\right)\right)=b^{-1}\left(r_{1}\right) \times_{\left(b / r_{1}\right)^{(1 / 2-v)}}\left[r_{1}, \infty\right) .
$$

Note that we are only prescribing the manifold's properties on one end or on a subset of that end, $b^{-1}\left(\left[r_{1}, \infty\right)\right)$, and we only prove that that end is rigid. The rest of the manifold can have larger volume growth and any kind of curvature.

When $v=1 / 2$, this theorem combined with [So2, Cor 23] and some simple calculations imply the following corollary.

Corollary 10. Let $M^{n}$ be a complete noncompact manifold with globally nonnegative Ricci curvature such that

$$
\lim _{r \rightarrow \infty} \frac{\operatorname{Vol}\left(B_{x_{0}}(r)\right)}{r}=\operatorname{Vol}_{n-1}\left(B_{x_{0}}\left(R_{0}\right) \cap b^{-1}\left(r_{1}\right)\right) .
$$

Then $M^{n}$ has only one end and that end is an isometric product manifold, $b^{-1}\left(r_{1}\right) \times\left[r_{1}, \infty\right)$ where $b^{-1}\left(r_{1}\right) \subset \bar{B}_{x_{0}}\left(R_{0}\right)$.

It is important to note that the Busemann function, in general, is not a smooth function, although its gradient is 1 almost everywhere. In order to prove that the level sets are smooth on a manifold with strongly minimal volume growth, we will show that the Busemann function satisfies the following elliptic partial differential equation in the weak sense:

$$
\Delta b=\frac{(n-1)\left(\frac{1}{2}-v\right)}{b} .
$$

This equation is satisfied by the function $b$ on a manifold with a metric of the form

$$
d b^{2}+\left(\frac{b}{r_{0}}\right)^{\left(\frac{1}{2}-v\right) 2} g_{0}
$$


Recall that we plan to prove that the end, $b^{-1}\left(\left[r_{1}, \infty\right)\right)$ has a metric of this form. We can then use elliptic regularity to obtain smoothness.

We first show that manifolds with strongly minimal volume growth have proper Busemann functions and the ratios, $\operatorname{Vol}_{n-1}\left(b^{-1}\left(\left[r_{2}, r_{3}\right]\right)\right) /\left(r_{3}^{p}-r_{2}^{p}\right)$, are constant with respect to $r_{2}$ and $r_{3}$ [Lemmas 11 and 12]. We then compare these regions to subsets of annuli about $\gamma(R)$ using the fact that the Busemann function's level sets are compact [Defn 13, Lemma 14]. Recall that

$$
b(x)=\lim _{R \rightarrow \infty} R-\rho_{R}(x) \text { where } \rho_{R}(x)=d(x, \gamma(R)) .
$$

We then employ the Relative Volume Comparison Theorem of $[\mathrm{BiCr}]$ and [GrLaPa] combined with the volume estimate on these regions, to control $\Delta \rho_{R}$ in a weak sense [Lemma 18]. Then taking $R$ to infinity we show that $b(x)$ satisfies the elliptic equation (1.7) in a weak sense [Lemma 20]. Once we have proven that the Busemann function is smooth, we use the BochnerWeitzenboch Formula to prove that the metric is rigid [Lemma 1.53].

Lemma 11. For $M^{n}$ as above, let $K=\bar{B}_{x_{0}}\left(R_{0}\right) \cap b^{-1}\left(\left(-\infty, r_{1}\right]\right)$. Then

$$
b^{-1}\left(\left[r_{1}, \infty\right)\right) \subset \Omega(K)
$$

and thus the Busemann function, $b$, is proper.

Proof. By [So2, Lemma 4], $\Omega(K) \cap b^{-1}\left(\left[r_{1}, \infty\right)\right)$ is a closed set. Thus if there exists a point in $b^{-1}\left(\left[r_{1}, \infty\right)\right)$ which is not in $\Omega(K)$ then there exists a ball

$$
B_{q}(\delta) \subset b^{-1}\left(\left[r_{1}, \infty\right)\right) \backslash \Omega(K) .
$$

Let $s=b(q)+\delta / 2$ and $t=b(q)-\delta$.

Let $U=b^{-1}([t, s]) \cap \bar{B}_{q}(\delta)$. Thus

$$
\operatorname{Vol}_{n-1}\left(\Omega(\bar{U}) \cap b^{-1}(s)\right) \geq \operatorname{Vol}_{n-1}\left(\bar{B}_{q}(\delta) \cap b^{-1}(s)\right)>0 .
$$

Applying Theorem 5 we have,

$$
\operatorname{Vol}\left(\Omega(\bar{U}) \cap b^{-1}([s, r])\right) \geq \frac{r^{p}-s^{p}}{p s^{p-1}} \operatorname{Vol}_{n-1}\left(\Omega(\bar{U}) \cap b^{-1}(s)\right)
$$

Let $r_{2}$ be a real number such that

$$
B_{x_{0}}\left(r_{2}\right) \supset\left(\Omega(\bar{U}) \cap b^{-1}(s)\right) \cup\left(\Omega(K) \cap b^{-1}(s)\right) .
$$


By the definition of $\Omega(K)$ [Defn 4], for all $p \in \Omega(K) \cap b^{-1}([s, t])$ there exists $z \in K$ such that $\gamma_{z}(b(p))=p$. So,

$$
d\left(p, \Omega(K) \cap b^{-1}(s)\right) \leq b(p)-s \leq t-s .
$$

Thus,

$$
\left.\left.B_{x_{0}}\left(r+r_{2}-s\right) \cap b^{-1}\left(\left[r_{1}, \infty\right)\right) \supset\left(\Omega(K) \cap b^{-1}(s, r)\right)\right) \cup\left(\Omega(\bar{U}) \cap b^{-1}(s, r)\right)\right)
$$

where the union is disjoint. So by Theorem 5 we have,

$$
\begin{aligned}
\lim _{r \rightarrow \infty} \frac{\operatorname{Vol}\left(B_{x_{0}}\left(r+r_{2}-s\right) \cap b^{-1}\left(\left[r_{1}, \infty\right)\right)\right)}{r^{p}-s^{p}} \geq \\
\geq \frac{V o l\left(\Omega(K) \cap b^{-1}\left(r_{0}, s\right)\right)}{s^{p}-r_{0}^{p}}+\frac{V o l_{n-1}\left(\Omega(\bar{U}) \cap b^{-1}((s))\right)}{p s^{p-1}} .
\end{aligned}
$$

However, by the strongly minimal volume growth, we have

$$
\begin{aligned}
\lim _{r \rightarrow \infty} \frac{\operatorname{Vol}\left(B_{x_{0}}\left(r+r_{2}-s\right) \cap b^{-1}\left(\left[r_{1}, \infty\right)\right)\right)}{r^{p}-s^{p}} & =\frac{V o l_{n-1}\left(B_{x_{0}}\left(R_{0}\right) \cap b^{-1}\left(r_{1}\right)\right)}{p r_{1}^{p}} \\
& \leq \frac{V o l\left(\Omega(K) \cap b^{-1}\left(r_{1}, s\right)\right)}{s^{p}-r_{1}^{p}},
\end{aligned}
$$

and so,

$$
\operatorname{Vol}_{n-1}\left(\Omega(\bar{U}) \cap b^{-1}((s))\right)=0,
$$

which contradicts (1.9).

Thus

$$
b^{-1}\left(\left[r_{1}, \infty\right)\right) \subset \Omega(K) .
$$

This implies that $b^{-1}(r) \subset B_{x_{0}}\left(r-r_{1}+R_{0}\right)$ for any $r \geq r_{1}$, so it is compact.

Furthermore, for $r \leq r_{1}, b^{-1}(r)$ is a subset of the closed tubular neighborhood $T_{r_{1}-r}\left(b^{-1}\left(r_{1}\right)\right)$ as can be seen by using Busemann rays to travel from $b^{-1}(r)$ to $b^{-1}\left(r_{1}\right)$. Thus $b$ is a proper function.

Lemma 12. Given $M^{n}$ as described above, then the ratio

$$
V(r)=\frac{V o l_{n-1}\left(\Omega(K) \cap b^{-1}(r)\right)}{r^{\left(\frac{1}{2}-v\right)(n-1)}}
$$

is a constant function for all $r \geq r_{1}$, and, thus,

$$
\frac{V o l_{n-1}\left(b^{-1}\left(\left[r_{2}, r_{3}\right]\right)\right)}{\left(r_{3}^{p}-r_{2}^{p}\right)}=\frac{V o l_{n-1}\left(b^{-1}\left(r_{1}\right) \cap \bar{B}_{x_{0}}\left(R_{0}\right)\right)}{p r_{1}^{p-1}}
$$

for all $r_{3}>r_{2} \geq r_{1}$. 
Proof. Let $K=\bar{B}_{x_{0}}\left(R_{0}\right) \cap b^{-1}\left(r_{1}\right)$ as in Lemma 11. By the definition of $\Omega(K)$, we know that for all $x \in \Omega(K) \cap b^{-1}\left(r_{1}, r\right)$ there exists $x^{\prime} \in K \subset$ $\bar{B}_{x_{0}}\left(R_{0}\right)$ such that $x \in \gamma_{x^{\prime}}\left(\left[r_{1}, r\right]\right)$; so

$$
\Omega(K) \cap b^{-1}\left(\left(r_{1}, r\right)\right) \subset \bar{B}_{x_{0}}\left(r-r_{1}+R_{0}\right) .
$$

Note also that

$$
K \cap b^{-1}\left(r_{1}\right)=\Omega(K) \cap b^{-1}\left(r_{1}\right),
$$

for our choice of $K$. Strongly minimal volume growth, Theorem 5, (1.15) and (1.16) imply that for any $b>a \geq r_{1}$

$$
\begin{aligned}
\frac{V o l_{n-1}\left(K \cap b^{-1}\left(r_{1}\right)\right)}{p r_{1}^{p-1}} & =\lim _{r \rightarrow \infty} \frac{V o l\left(B_{x_{0}}\left(r-r_{1}+R_{0}\right)\right) \cap b^{-1}\left(\left[r_{1}, \infty\right)\right)}{r^{p}-r_{1}^{p}} \\
& \geq \lim _{r \rightarrow \infty} \frac{V o l\left(\Omega(K) \cap b^{-1}\left(r_{1}, r\right)\right)}{r^{p}-r_{1}^{p}} \\
& \geq \frac{V o l_{n-1}\left(K \cap b^{-1}\left(r_{1}\right)\right)}{p r_{1}^{p-1}}
\end{aligned}
$$

Since all the inequalities must be equalities and the limit in (1.18) is monotone, we get

$$
\frac{V o l_{n-1}\left(K \cap b^{-1}\left(r_{1}\right)\right)}{p r_{1}^{p-1}}=\frac{\operatorname{Vol}\left(\Omega(K) \cap b^{-1}\left(r_{1}, r\right)\right)}{r^{p}-r_{1}^{p}},
$$

for any $r>r_{1}$. Subtracting (1.20) with $r=a$ from (1.20) with $r=b$, and reworking the equation gives

$$
\frac{\operatorname{Vol}\left(\Omega(K) \cap b^{-1}(a, b)\right)}{b^{p}-a^{p}}
$$

is a constant with respect to $a$ and $b$. Then applying Lemma 11, we obtain the lemma.

The fact that the Busemann function is proper in a manifold with strongly minimal volume growth makes it much easier to prove that the metric is rigid than if this were not the case. We need not trace through the rather involved proof of Theorem 5 with its unions of star-shaped sets about points in $b^{-1}(R)$ [So2]. Instead we use the following very simple sets.

First we fix $r_{3}>r_{1} \geq r_{0}$. We will prove that $b^{-1}\left(\left[r_{1}, r_{3}\right]\right)$ is isometric to the appropriate warped product manifold $b^{-1}\left(r_{1}\right) \times_{\left(b / r_{1}\right)^{(1 / 2-v)}}\left[r_{1}, r_{3}\right]$ and that $b$ is smooth on $b^{-1}\left(\left[r_{1}, r_{3}\right]\right)$. To do so we fix $\varepsilon_{0}<\left(r_{3}-r_{1}\right) / 10$ and take $r_{4}=2 r_{3}$. 
Definition 13. Let $R>r_{4}$ and $r_{1} \leq a<b \leq R$ we define the inner annulus,

$$
S_{a, b, R}^{r_{4}}=A n n_{R-b, R-a}(\gamma(R)) \cap S_{\gamma(R)}^{r_{4}}
$$

where

$S_{\gamma(R)}^{r_{4}}=\left\{\sigma([0, L]): \sigma\right.$ is a min geod s.t. $\left.\sigma(0)=\gamma(R), \sigma(L) \in b^{-1}\left(\left(-\infty, r_{4}\right]\right)\right\}$.

We will often write only $S_{a, b, R}=S_{a, b, R}^{r_{4}}$.

Recall that $b(x)=\lim _{R \rightarrow \infty}\left(R-\rho_{R}(x)\right)$ where the convergence is uniform on compact sets. So given any $\varepsilon>0$ and any compact set like $b^{-1}\left(\left[r_{1}, r_{4}\right]\right)$, there exists $R_{\varepsilon}=R_{\varepsilon}^{r_{1}, r_{4}}$ such that

$$
b(x) \leq R-\rho_{R}(x)+\varepsilon \quad \forall R \geq R_{\varepsilon} .
$$

On the other hand, by the definition of $b(x)$, we have

$$
b(x) \geq \lim _{s \rightarrow \infty}(s-d(x, \gamma(R))-d(\gamma(R), \gamma(s)))=R-d(x, \gamma(R)) .
$$

Thus the inner annuli, $S_{a, b, R}$, are close to the compact regions $b^{-1}([a, b])$ as described precisely in the following lemma.

Lemma 14. Let $r_{1} \leq a<b \leq r_{4}$. Then for all $\varepsilon>0$ there exists $R_{\varepsilon}=$ $R_{\varepsilon}^{r_{1}, r_{4}}$ as defined in (1.23) such that

$$
S_{a+\varepsilon, b-\varepsilon, R} \subset b^{-1}([a, b]) \subset S_{a-\varepsilon, b+\varepsilon, R}
$$

for all $R \geq R_{\varepsilon}$.

Proof. Fix $R \geq R_{\varepsilon}$.

Suppose $x \in b^{-1}([a, b]) \subset b^{-1}\left(\left[r_{1}, r_{4}\right]\right)$. Then by (1.23) and (1.24), we have

$$
L_{x}=\rho_{R}(x) \in[R-b-\varepsilon, R-a+\varepsilon] .
$$

Thus there exists a minimal geodesic $\sigma$ from $\sigma(0)=\gamma(R)$ to $\sigma\left(L_{x}\right)=x \in$ $b^{-1}\left(\left[r_{1}, r_{4}\right]\right)$. So

$$
x \in S_{a-\varepsilon, b+\varepsilon, R} .
$$

On the other hand, if $y \in S_{a+\varepsilon, b-\varepsilon, R}$, then there exists $L>0$ and a minimal geodesic, $\sigma$, from $\sigma(0)=\gamma(R)$ to $\sigma(L) \in b^{-1}\left(\left[r_{1}, r_{4}\right]\right)$ and there 
exists $t \in[R-b+\varepsilon, \min \{R-a-\varepsilon, L\}]$ such that $y=\sigma(t)$. Since $\sigma(L) \in$ $b^{-1}\left(\left[r_{1}, r_{4}\right]\right)$, we can apply (1.23) to get,

$$
b(\sigma(L)) \leq R-L+\varepsilon
$$

Thus

$b(y) \leq b(\sigma(L))+d(\sigma(L), y)=b(\sigma(L))+L-d(y, \gamma(R)) \leq R+\varepsilon-(R-b+\varepsilon)=b$.

Meanwhile, by (1.24) we have,

$$
b(y) \geq R-d(y, \gamma(R)) \geq R-(R-a-\varepsilon)>a
$$

and we have $y \in b^{-1}([a, b])$.

Thus the volumes of the regions between Busemann level sets can be compared to the volumes of these inner annuli. On the other hand, the volumes of the inner annuli can be controlled using the Relative Volume Comparison Theorem on the star-shaped sets, $S_{r_{1}, R, R}$ about $\gamma(R)[\mathrm{BiCr}$, $\mathrm{GrLaPa}, \mathrm{Ch}]$. We can bound the Ricci curvature from below using the following lemma which is a direct consequence of the techniques used in the last proof.

Lemma 15. Given any $\varepsilon$, let $\varepsilon_{v}=\varepsilon$ if $v<1 / 2$ and $\varepsilon_{v}=0$ otherwise.

There exists $R_{\varepsilon}^{r_{1}, r_{4}}$ as defined in (1.23) such that for all $x$ in the star shaped set, $S_{r_{1}, R, R}^{r_{1}, r_{4}}$, we have

$$
R i c_{y} \geq \frac{(n-1)\left(\frac{1}{4}-v^{2}\right)}{\left(R-d(y, \gamma(R))+\varepsilon_{v}\right)^{2}}
$$

for all $R \geq R_{\varepsilon}$.

Thus we have the same lower Ricci curvature bound as the comparison manifolds of Definition 6 if we consider $\gamma(R)$ to be the base point.

We will now control the mean curvature of the level sets of $\rho_{R}$ within $S_{r_{1}, R, R}$ using the comparison manifolds, $M_{R, \varepsilon}^{n}$, of Definition 6 combined with the above lemma regarding Ricci curvature and the Relative Volume Comparison Theorem. To control the mean curvature from below we will employ the volume estimates on the regions between Busemann levels and the relationship between those regions and the inner annuli. We begin with some facts and definitions regarding the mean curvature of a distance function. 
Definition 16. Let $m_{R}(x)$ be the mean curvature with respect to the inward normal of the sphere of radius $d(x, \gamma(R))$ about $\gamma(R)$ evaluated at $x$. Recall that this mean curvature is well defined along minimal geodesics to $\gamma(R)$ and is equal to $\Delta \rho(x)$ where it is defined. See, for example, [Ch].

Given $x \in M^{n}$, let $\bar{m}_{R, \varepsilon}(x)$ be the mean curvature with respect to the inward normal of the distance sphere of radius $\rho_{R}(x)$ about the origin in the comparison warped product manifold, $M_{R, \varepsilon}^{n}$.

Lemma 17. The comparison mean curvature satisfies

$$
\bar{m}_{R, \varepsilon}(x)=\frac{-(n-1)}{R+\varepsilon-\rho_{R}(x)}\left(\frac{1}{2}+v \operatorname{coth}\left(\log \left(\frac{R+\varepsilon-\rho_{R}(x)}{R+\varepsilon}\right)\right)\right)
$$

and as $R$ goes to infinity, we have

$$
\lim _{R \rightarrow \infty} \bar{m}_{R, \varepsilon}(x)=\frac{-(n-1)}{b(x)}\left(\frac{1}{2}+v(-1)\right)
$$

uniformly on compact sets.

The proof of the lemma is an exercise on warped product manifolds and can be found in [So1].

We now need to show that the mean curvature of spheres around $\gamma(R)$, $m_{R}(x)$ approaches $\bar{m}_{\varepsilon, R}(x)$ as $R$ approaches infinity and $\varepsilon$ goes to 0 . Then, in some weak sense we will have

$$
\Delta b(x)=-\lim _{R \rightarrow \infty} \Delta \rho(x)=-\lim _{R \rightarrow \infty} m_{R}(x)=\frac{-(n-1)(1 / 2-v)}{b(x)}
$$

Since $\Delta \rho_{R}(x)$ and $\Delta b(x)$ are defined on different subsets of $M$, we need to prove (1.28) very carefully. In the next lemma we will only obtain a weak estimate on $\lim _{R \rightarrow \infty} m_{R}(x)$ using the strongly minimal volume growth, but that will suffice.

Lemma 18. Let $M^{n}$ have the properties defined at the beginning of this section. Fix $r_{3}>r_{1}$. Then for all $\delta>0$, there exists $\varepsilon_{\delta}>0$ such that for all $\varepsilon<\varepsilon_{\delta}$ there exists $R_{\delta}\left(\varepsilon, r_{1}, r_{3}\right)>2 r_{3}$ and there exists a constant, $C_{r_{1}, r_{3}, R+\varepsilon}$, such that $\lim _{R \rightarrow \infty} C_{r_{1}, r_{3}, R+\varepsilon}=C_{r_{1}, r_{3}}>0$ such that for all $R \geq R_{\delta}\left(\varepsilon, r_{1}, r_{3}\right)$, we have,

$$
0<\int_{s=r_{1}}^{r_{3}} \int_{S_{s, r_{3}, R}(K)}\left(\Delta \rho_{R}(x)-\bar{m}_{R, \varepsilon}(x)\right) d v o l d t<\frac{\delta}{C_{r_{1}, r_{3}, R+\varepsilon}} .
$$

Furthermore, the integrand is nonnegative. 
In this lemma $d v o l$ is the volume form on $M^{n}$. The integral over $S_{a, b, R}(K)$ can be best understood using the following definition. Recall Definition 13.

Definition 19. Let $\Theta(R) \subset T M_{\gamma(R)}$ be defined as

$$
\begin{aligned}
\Theta_{R} & =\Theta_{R}^{r_{4}}= \\
& =\left\{\sigma^{\prime}(0): \sigma \text { is a min geod s.t. } \sigma(0)=\gamma(R), \sigma(L) \in b^{-1}\left(\left(-\infty, r_{4}\right]\right)\right\},
\end{aligned}
$$

Given any $\theta \in \Theta_{R}$, let $A_{R}(t, \theta)$ be the warped product of the Jacobi fields along $\exp _{\gamma(R)} t \theta$ which are 0 when $t=0$ and whose first covariant derivatives are orthonormal when $t=0$. We set $A_{R}(t, \theta)$ to be continuous up to and including the cut point. After a cut point it is set to be 0 .

So $d v o l=A_{R}(t, \theta) \nu_{\theta} d t$ where $\nu_{\theta}$ is the volume form on the unit sphere.

Proof of Lemma 18. Let $r_{4}=2 r_{3}>r_{3}$. Let $S_{a, b, R}=S_{a, b, R}^{r_{4}}$ as in Definition 13.

Given any $\varepsilon>0$ and $R>r_{3}$, let

$$
J(t)=J_{\varepsilon_{v}, R}(t)
$$

be the Jacobi field defined in Definition 6 where $\varepsilon_{v}=\varepsilon$ if $v<1 / 2$ and $\varepsilon_{v}=0$ otherwise. By the Bishop Volume Comparison Theorem [BiCr], the lower bound on Ricci curvature in the comparison manifolds, (1.4), and the Ricci curvature bound on $S_{a, b, R}$, [Lemma 15], we know that $\frac{A_{R}(t, \theta)}{J(t)^{n-1}}$ is nonincreasing for $t \in\left[0, R-r_{1}\right]$ and $\theta \in \Theta_{R}$. We wish to show that this ratio is almost constant.

First we note that, by Definition 19 and (1.24), if $a \leq b \leq r_{4}$ and $\theta \in \Theta_{R}$ such that $\exp _{\gamma(R)}(t \theta)$ is minimal for $t \in[0, R-a]$, then

$$
b\left(\exp _{\gamma(R)}((R-a) \theta)\right) \leq R-(R-a) \leq r_{4} .
$$

So by Definition $13, \exp _{\gamma(R)}(t \theta) \in S_{a, b, R}$ for all $t \in[R-b, R-a]$ and we have

$$
\operatorname{Vol}\left(S_{a, b, R}\right)=\int_{R-b}^{R-a} \int_{\Theta_{R}} A_{R}(t, \theta) d \nu_{\theta} d t
$$

Thus, by the Relative Volume Comparison Theorem,

$$
\frac{V o l\left(S_{r_{3}, r_{4}, R}\right)}{\operatorname{Vol}\left(S_{r_{1}, r_{3}, R}\right)}=\frac{\int_{R-r_{4}}^{R-r_{3}} \int_{\theta_{R}} A_{R}(t, \theta) d \nu_{\theta} d t}{\int_{R-r_{3}}^{R-r_{1}} \int_{\theta_{R}} A_{R}(t, \theta) d \nu_{\theta} d t} \geq \frac{\int_{R-r_{4}}^{R-r_{3}} J_{R . \varepsilon}(t)^{n-1} d t}{\int_{R-r_{3}}^{R-r_{1}} J_{R . \varepsilon}(t)^{n-1} d t}
$$


By Lemma 14, we know that for any $\varepsilon>0$, there exists $R_{\varepsilon}$ such that

$$
\frac{\operatorname{Vol}\left(S_{r_{3}, r_{4}, R}\right)}{\operatorname{Vol}\left(S_{r_{1}, r_{3}, R}\right)} \leq \frac{\operatorname{Vol}\left(b^{-1}\left(\left[r_{3}-\varepsilon, r_{4}+\varepsilon\right]\right)\right)}{\operatorname{Vol}\left(b^{-1}\left(\left[r_{1}+\varepsilon, r_{3}-\varepsilon\right]\right)\right)} \quad \forall R \geq R_{\varepsilon} .
$$

By the strongly minimal volume growth and Lemma 12, we have

$$
\frac{\operatorname{Vol}\left(b^{-1}\left(\left[r_{3}-\varepsilon, r_{4}+\varepsilon\right]\right)\right)}{\operatorname{Vol}\left(b^{-1}\left(\left[r_{1}+\varepsilon, r_{3}-\varepsilon\right]\right)\right)}=\frac{\left(r_{4}+\varepsilon\right)^{p}-\left(r_{3}-\varepsilon\right)^{p}}{\left(r_{3}-\varepsilon\right)^{p}-\left(r_{1}+\varepsilon\right)^{p}}
$$

On the other hand, by (1.5),

$$
\lim _{R \rightarrow \infty} \frac{\int_{R-r_{4}}^{R-r_{3}} J_{R . \varepsilon}(t)^{n-1} d t}{\int_{R-r_{3}}^{R-r_{1}} J_{R . \varepsilon}(t)^{n-1} d t}=\frac{\left(r_{4}\right)^{p}-\left(r_{3}\right)^{p}}{\left(r_{3}\right)^{p}-\left(r_{1}\right)^{p}}
$$

Given any $\delta^{\prime}>0$, there exists $\varepsilon_{\delta^{\prime}}>0$ small enough that the right hand side of (1.35) is within $\delta^{\prime}$ of the right hand side of (1.34) for all $\varepsilon<\varepsilon_{\delta^{\prime}}$, and there exists $\bar{R}_{\delta^{\prime}}$ large enough that the the right hand side of (1.35) is within $\delta^{\prime}$ of the right hand side of (1.32). Thus the inequalities in (1.32)-(1.35) are almost equalities, and we have the following.

For all $\delta>0$, let $\delta^{\prime}=\delta /\left(2 \operatorname{Vol}\left(S_{r_{1}, r_{3}, R}\right)\right)$, there exists $\varepsilon_{\delta^{\prime}}>0$, such that for all $\varepsilon<\varepsilon_{\delta^{\prime}}$, there exists

$$
R_{\delta}\left(\varepsilon, r_{1}, r_{3}\right)=\max \left\{R_{\varepsilon}, \bar{R}_{\delta^{\prime}}\right\}
$$

such that we have

$$
0 \leq \frac{\int_{R-r_{4}}^{R-r_{3}} \int_{\theta_{R}} A_{R}(t, \theta) d \nu_{\theta} d t}{\int_{R-r_{3}}^{R-r_{1}} \int_{\theta_{R}} A_{R}(t, \theta) d \nu_{\theta} d t}-\frac{\int_{R-r_{4}}^{R-r_{3}} J_{R . \varepsilon}(t)^{n-1} d t}{\int_{R-r_{3}}^{R-r_{1}} J_{R . \varepsilon}(t)^{n-1} d t} . \leq 2 \delta^{\prime} .
$$

By multiplying both sides of (1.37) by $\operatorname{Vol}\left(S_{r_{1}, r_{3}, R}\right)$ and multiplying both sides by $\int_{R-r_{3}}^{R-r_{1}} J_{R . \varepsilon}(t)^{n-1} d t$, we have,

$$
\begin{aligned}
0 \leq & \int_{R-r_{4}}^{R-r_{3}} \int_{\theta_{R}} A_{R}(t, \theta) d \nu_{\theta} d t \int_{R-r_{3}}^{R-r_{1}} J(t)^{n-1} d t \\
& -\int_{R-r_{3}}^{R-r_{1}} \int_{\theta_{R}} A_{R}(t, \theta) d \nu_{\theta} d t \int_{R-r_{4}}^{R-r_{3}} J(t)^{n-1} d t \\
& \leq \delta \int_{R-r_{4}}^{R-r_{3}}(J(t))^{n-1} d t .
\end{aligned}
$$

As mentioned above, $\frac{A_{R}(t, \theta)}{J(t)^{n-1}}$ is nonincreasing. We wish to show that this ratio is almost constant using (1.38). 
Note that,

$$
\int_{R-b}^{R-a} \int_{\Theta_{R}} A_{R}(t, \theta) d \nu_{\theta} d t=\int_{R-r}^{R-r_{3}} \int_{\Theta_{R}} \frac{A_{R}(t, \theta)}{J(t)^{n-1}} J(t)^{n-1} d \nu_{\theta} d t .
$$

By substituting this into (1.38) and by subtracting and adding

$$
\int_{\Theta_{R}} \frac{A_{R}\left(R-r_{3}, \theta\right)}{J\left(R-r_{3}\right)^{n-1}} d \nu_{\theta} \int_{R-r_{3}}^{R-r_{1}} J(t)^{n-1} d t \int_{R-r}^{R-r_{3}} J(t)^{n-1} d t
$$

to (1.38), we get

$$
\delta \int_{R-r_{4}}^{R-r_{3}} J(t)^{n-1} d t>I_{1} \int_{R-r_{3}}^{R-r_{1}} J(t)^{n-1} d t+I_{2} \int_{R-r_{4}}^{R-r_{3}} J(t)^{n-1} d t
$$

where

$$
I_{1}=\int_{R-r_{4}}^{R-r_{3}} \int_{\Theta_{R}}\left(\frac{A_{R}(t, \theta)}{J(t)^{n-1}}-\frac{A_{R}\left(R-r_{3}, \theta\right)}{J\left(R-r_{3}\right)^{n-1}}\right) J(t)^{n-1} d \nu_{\theta} d t
$$

and

$$
I_{2}=\int_{R-r_{3}}^{R-r_{1}} \int_{\Theta_{R}}\left(\frac{-A_{R}(t, \theta)}{J(t)^{n-1}}+\frac{\left.A_{R}\left(R-r_{3}, \theta\right)\right)}{J\left(R-r_{3}\right)^{n-1}}\right) J(t)^{n-1} d \nu_{\theta} d t
$$

Since $\frac{A_{R}(t)}{J(t)^{n-1}}$ is decreasing, the integrands of $I_{1}$ and $I_{2}$ are both positive. Thus, $I_{2}<\delta$ and

$$
0<\int_{R-r_{3}}^{R-r_{1}} \int_{\Theta_{R}}\left|\frac{A_{R}\left(R-r_{3}, \theta\right)}{\left(J\left(R-r_{3}\right)\right)^{n-1}}-\frac{A_{R}(t, \theta)}{(J(t))^{n-1}}\right| J(t)^{n-1} d \nu_{\theta} d t<\delta
$$

Thus the ratio, $\frac{A_{R}(t, \theta)}{J(t)^{n-1}}$, is almost constant.

Given $\theta \in \Theta_{R}$, let $d_{\theta} \in\left(R-r_{4}, \infty\right]$ be the distance to the cutpoint of $\gamma(R)$ along $\exp _{\gamma(R)}(t \theta)$. Then $A_{R}(t, \theta)$ is smooth for $t \in\left(0, d_{\theta}\right)$, continuous for $t \in\left[0, d_{\theta}\right]$, and 0 for $t>d_{\theta}$. Since the mean curvatures are evaluated with respect to the inward normal, we also know that

$$
\frac{A_{R}^{\prime}(t, \theta)}{A_{R}(t, \theta)}=-m_{R}\left(\exp _{\gamma}(R)(t \theta)\right)=-\Delta \rho_{R}\left(\exp _{\gamma}(R)(t \theta)\right),
$$

for $t \in\left(0, d_{\theta}\right)$, where $A_{R}^{\prime}(t, \theta)=\frac{\partial}{\partial t} A_{R}(t, \theta)$. See, for example [Ch]. 
Let $r_{\theta, t}=\max \left\{\min \left\{t, d_{\theta}\right\}, R-r_{3}\right\}$. We will use this function to seperate off the differentiable part of the integrand of (1.42).

$$
\begin{aligned}
0< & \int_{R-r_{3}}^{R-r_{1}} \int_{\Theta_{R}}\left(\frac{A_{R}\left(R-r_{3}, \theta\right)}{J^{n-1}\left(R-r_{3}\right)}-\frac{A_{R}\left(r_{\theta, t}, \theta\right)}{J^{n-1}\left(r_{\theta, t}\right)}\right) J^{n-1}\left(r_{\theta, t}\right) d \nu_{\theta} d t \\
& +\int_{R-r_{3}}^{R-r_{1}} \int_{\Theta_{R}}\left(\frac{A_{R}\left(r_{\theta, t}, \theta\right)}{J^{n-1}(t)}-\frac{A_{R}(t, \theta)}{J^{n-1}(t)}\right) J^{n-1}\left(r_{\theta, t}\right) d \nu_{\theta} d t<\delta .
\end{aligned}
$$

Since $t \geq r_{\theta, t} \geq R-r_{3}$ and the ratios are decreasing, both integrands are positive. Thus we have,

$$
0<\int_{t=R-r_{3}}^{R-r_{1}} \int_{\Theta_{R}}\left(\frac{A_{R}\left(R-r_{3}, \theta\right)}{J^{n-1}\left(R-r_{3}\right)}-\frac{A_{R}\left(r_{\theta, t}, \theta\right)}{J^{n-1}(t)}\right) J^{n-1}(t) d \nu_{\theta} d t<\delta .
$$

The function $A_{R}$ is differentiable in this integral, so

$$
0<\int_{t=R-r_{3}}^{R-r_{1}} \int_{\Theta_{R}}\left(-\int_{l=R-r_{3}}^{r_{\theta, t}} \frac{d}{d l}\left(\frac{A_{R}(l, \theta)}{J^{n-1}(l)}\right) d l\right) J^{n-1}(t) d \nu_{\theta} d t<\delta,
$$

and

$-\int_{t=R-r_{3}}^{R-r_{1}} \int_{\Theta_{R}} \int_{l=R-r_{3}}^{r_{\theta, t}}\left(\frac{A_{R}^{\prime}(l, \theta)}{A_{R}(l, \theta)}-\frac{\left(J^{n-1}(l)\right)^{\prime}}{J^{n-1}(l)}\right) \frac{J^{n-1}(t)}{J^{n-1}(l)} A_{R}(l, \theta) d l d \nu_{\theta} d t<\delta$.

Recall that $S_{R-t, r_{3}, R}$ only consists of points on minimizing geodesics from $\gamma(R)$ to $b^{-1}\left(\left(-\infty, r_{4}\right]\right)$. Thus

$$
S_{R-t, r_{3}, R}=\left\{\exp _{\gamma(R)}(l \theta): \theta \in \Theta_{R}, l \in\left[R-r_{3}, r_{\theta, t}\right)\right\}
$$

and $\exp _{\gamma(R)}$ is invertible on $S_{R-t, r_{3}, R}$. Let $l=\rho_{R}(x)$ and $\theta$ be defined such that $x=\exp _{\gamma(R)}(l \theta)$ where $x \in S_{R-t, r_{3}, R}$. Furthermore $d v o l=$ $A_{R}(l, \theta) d l d \nu_{\theta}$ on $S_{R-t, r_{3}, R}$.

So we have

$$
0<-\int_{t=R-r_{3}}^{R-r_{1}} \int_{S_{R-t, r_{3}, R}}\left(\frac{A_{R}^{\prime}(l, \theta)}{A_{R}(l, \theta)}-\frac{\left(J^{n-1}(l)^{\prime}\right.}{J^{n-1}(l)}\right) d v o l d t<\frac{\delta}{C_{r_{1}, r_{3}, R+\varepsilon_{v}}}
$$

where

$$
C_{r_{1}, r_{3}, R+\varepsilon_{v}}=\min _{\left(R-r_{1} \geq t \geq l \geq R-r_{3}\right)}\left(\frac{J_{R, \varepsilon_{v}}^{n-1}(t)}{J_{R, \varepsilon_{v}}^{n-1}(l)}\right) .
$$


Since this integral avoids cut points of $\gamma(R)$, we have

$$
0<-\int_{t=R-r_{3}}^{R-r_{1}} \int_{S_{R-t, r_{3}, R}}\left(-\Delta \rho_{R}(x)+\bar{m}_{R, \varepsilon}(x)\right) d v o l d t<\frac{\delta}{C_{r_{1}, r_{3}, R+\varepsilon}} .
$$

Setting $s=R-t$, we are done proving (1.29) with $R_{\delta}\left(\varepsilon, r_{1}, r_{3}\right)$ defined in (1.36).

Finally, for all $v \in\left[0, \frac{n+1}{2(n-1)}\right]$, we know that

$$
\begin{aligned}
\lim _{R \rightarrow \infty} C_{r_{1}, r_{3}, R+\varepsilon} & =\lim _{R \rightarrow \infty} \min _{r_{1} \leq t^{\prime} \leq l^{\prime} \leq r_{3}} \frac{-\left(t^{\prime}+\varepsilon\right)^{v+1 / 2}+(R+\varepsilon)^{2 v}\left(t^{\prime}+\varepsilon\right)^{-v+1 / 2}}{-\left(l^{\prime}+\varepsilon\right)^{v+1 / 2}+(R+\varepsilon)^{2 v}\left(l^{\prime}+\varepsilon\right)^{-v+1 / 2}} \\
& =\min _{r_{1} \leq t^{\prime} \leq l^{\prime} \leq r_{3}} \frac{\left(t^{\prime}+\varepsilon\right)^{-v+1 / 2}}{\left(l^{\prime}+\varepsilon\right)^{-v+1 / 2}}>0 .
\end{aligned}
$$

In Lemma 18 , the estimate on $\Delta \rho_{R}(x)$ involves a double integral. This can be studied as a single integral over a region in the isometric product, $M \times \mathbb{R}$, where we define the extended Busemann function, $\bar{b}(x, s)=b(x)$, and the extended distance function, $\bar{\rho}_{R}(x, s)=\rho(x)$. Note that the Laplacians of these extended functions on $M \times \mathbb{R}$ are the same as the original functions' Laplacians in $M$ because the extended functions are constant in $s$.

Proposition 20. Let $M^{n}$ be as defined above.

Then the Busemann function with respect to that ray, $b(x)$, is smooth and

$$
\Delta b(x)=\frac{(n-1)(1 / 2-v)}{b(x)}
$$

on $b^{-1}\left(\left(r_{1}, \infty\right)\right)$.

Proof. Choose any $x_{1} \in b^{-1}\left(\left(r_{1}, \infty\right)\right)$. Let $h=\frac{b\left(x_{1}\right)-r_{1}}{4}$, and let

$$
b_{1}=b\left(x_{1}\right)-h, b_{2}=b\left(x_{1}\right)+h, \text { and } r_{3}=b\left(x_{1}\right)+2 h .
$$

Define the open set, $U$, as follows:

$$
U=\left\{(x, s): s \in\left(b_{1}, b_{2}\right), x \in b^{-1}\left(s, b_{2}\right)\right\} \subset M \times \mathbb{R} .
$$


We will prove that

$$
\Delta \bar{b}(x, s)=\frac{(n-1)(1 / 2-v)}{\bar{b}(x, s)}
$$

in the weak sense on $U$.

By Lemma 14, there exists $R_{h}^{r_{1}, r_{3}}$ such that

$$
b^{-1}\left(\left[s, b_{2}\right]\right) \subset S_{s-h, b_{2}+h, R}^{2 r_{3}} \subset S_{s-h, r_{3}, R}^{2 r_{3}} .
$$

for all $R \geq R_{h}^{r_{1}, r_{3}}$. Thus, since $b_{1}-h>r_{1}$,

$$
U \subset\left\{(x, s): s \in\left(r_{1}, r_{3}\right), x \in S_{s, r_{3}, R}^{2 r_{3}}\right\} .
$$

Let $\phi: U \rightarrow \mathbb{R}$ be a smooth nonnegative function with compact support. By Lemma 18 and (1.47), we know that for all $\delta>0$ there exists $\varepsilon_{\delta}>0$, such that for all $\varepsilon<\varepsilon_{\delta}$, there exists $R_{\delta}\left(\varepsilon, r_{1}, r_{3}\right)$ and $C_{r_{1}, r_{2}, R+\varepsilon}$ as defined in Lemma 18, such that

$$
0<\int_{U} \phi(x, s)\left(\Delta \rho_{R}(x)-\bar{m}_{R, \varepsilon}(x)\right) d v o l d t<\frac{\delta}{C_{r_{0}, r_{1}, R+\varepsilon}}
$$

Here we have used the fact that the integrand of (1.29) is nonnegative and the integrand here is still nonnegative.

Note that $\Delta \rho_{R}(x)=-\Delta\left(R-\rho_{R}(x)\right)$ since $R$ is just a constant. Note also that $\rho_{R}(x)=\bar{\rho}_{R}(x, s)$. Using the fact that the cut off function, $\phi$, is 0 near the boundary to integrate by parts, we get

$$
\begin{aligned}
\int_{U} \phi & (x, s)\left(\Delta \rho_{R}(x)\right) d v o l d t= \\
& =\int_{U} \phi(x, s)\left(-\Delta\left(R-\bar{\rho}_{R}(x, s)\right)\right) d v o l d t \\
& =\int_{U}-\Delta \phi(x, s)\left(R-\bar{\rho}_{R}(x, s)\right) d v o l d t
\end{aligned}
$$

By the uniform convergence of $b(x)$ on $U \subset b^{-1}\left(\left[r_{1}, r_{3}\right]\right)$, we know there exists $R_{\delta, r_{1}, r_{3}}$ such that

$$
\left|R-\bar{\rho}_{R}(x, s)-\bar{b}(x, s)\right|<\delta \quad \forall R \geq R_{\delta, r_{1}, r_{3}} .
$$

Furthermore, by Lemma 17, there exists $R_{\delta}$ such that

$$
\left|\bar{m}_{R, \varepsilon}(x)-\frac{-(n-1)}{b(x)}\left(\frac{1}{2}+v(-1)\right)\right|<\delta \quad \forall R \geq R_{\delta}
$$


Substituting (1.49),(1.50) and (1.51) all into (1.48), for

$$
R>\max \left\{R_{\delta}, R_{\delta_{0}, r_{1}, r_{3}}, R_{\delta}\left(r_{1}, r_{3}\right)\right\}
$$

we have

$$
\int_{U}-\Delta \phi(x, s) \bar{b}(x, s)+\phi(x, s)\left(\frac{(n-1)\left(\frac{1}{2}-v\right)}{\bar{b}(x, s)}\right) d v o l d t<\frac{\delta}{C_{r_{0}, r_{1}}}+2 \delta V o l(U)
$$

where the integrand is nonnegative. This equation no longer depends on $R$ or $\varepsilon$, so it holds for all $\delta>0$. Taking $\delta$ to 0 we see that

$$
\Delta \bar{b}(x, s)=\frac{(n-1)(1 / 2-v)}{\bar{b}(x, s)}
$$

in the generalized sense on $U$.

By elliptic regularity, we know that $\bar{b}(x, s)$ is differentiable on $U$ and satisfies

$$
\Delta \bar{b}(x, s)=\frac{(n-1)(1 / 2-v)}{b(x)} .
$$

The fact that $\bar{b}(x, s)$ appears on both sides of (1.52) allows us to pull up its differentiability by its bootstraps as high as we want.

Note that

$$
B_{x_{1}}(h / 2) \times\left(b_{1}, b_{1}+h / 2\right) \subset U,
$$

so $b(x)=\bar{b}\left(x, b_{1}+h / 4\right)$ is smooth on $B_{x_{1}}(\varepsilon / 2)$ and satisfies the differential equation, (1.45). This can be done at each $x_{1} \in b^{-1}\left(\left(r_{1}, \infty\right)\right)$, so we have proven the proposition.

We can now use this proposition combined with the Bochner Weitzenboch formula to prove that $b^{-1}\left(\left(r_{1}, \infty\right)\right)$ is a warped product and thus the Busemann function is smooth on $b^{-1}\left(\left[r_{1}, \infty\right)\right)$.

Lemma 21. Let $M^{n}$ satisfy (0.1) with $v \in[0,(n+1) / 2(n-1))$. Suppose the Busemann function, $b(x)$, is a solution of

$$
\Delta b(x)=\frac{(n-1)}{b(x)}\left(\frac{1}{2}-(v)\right)
$$

on some subset, $b^{-1}\left(\left(r_{1}, \infty\right)\right) \subset b^{-1}\left(\left[r_{0}, \infty\right)\right)$,

Then $b^{-1}\left(\left[r_{1}, \infty\right)\right)$ is isomorphic to the warped product,

$$
b^{-1}\left(\left[r_{1}, \infty\right)\right)=b^{-1}\left(r_{1}\right) \times_{\left(b / r_{1}\right)^{(1 / 2-v)}}\left[r_{1}, \infty\right) .
$$


Proof. First, we write the Bochner Weitzenboch Formula applied to $\nabla b$. That is, we substitute $\nabla b=\xi$ into the formula,

$$
\frac{1}{2} \Delta\left(\xi^{i} \xi_{i}\right)=g^{r s} g^{a b} \xi_{a, r} \xi_{b, s}+g^{r s} g^{a b}\left(\xi_{a, r s}+\xi_{r, a s}-\xi_{r, s a}\right) \xi_{b}+R_{l m} \xi_{l} \xi_{m},
$$

of [Boc, Lemma 2], to get

$$
\frac{1}{2} \Delta|\nabla b|^{2}=\mid \text { Hess }\left.b\right|^{2}+\langle\nabla \Delta b, \nabla b\rangle+\langle\text { Ric } \nabla b, \nabla b\rangle .
$$

We now use the fact that $|\nabla b|=1$, the differential equation (1.53) and the Ricci bound to get

$$
0 \geq \mid \text { Hess }\left.b\right|^{2}+(1 / 2-(v))(n-1)<\nabla b^{-1}, \nabla b>+(n-1)\left(1 / 4-v^{2}\right) / b^{2} .
$$

Thus,

$0 \geq \mid$ Hess $\left.b\right|^{2}+(1 / 2-(v))(n-1)<-b^{-2} \nabla b, \nabla b>+(n-1)\left(1 / 4-v^{2}\right) b^{-2}$,

and

$$
\mid \text { Hess }\left.b\right|^{2} \leq(n-1) b^{-2}(1 / 2-v)(1-(1 / 2+v))=(n-1) b^{-2}(1 / 2-v)^{2} .
$$

On the other hand,

$$
\mid \text { Hess }\left.b\right|^{2}=\sum_{i \neq j} b_{i, j}^{2}+\sum_{i \neq 1} b_{i, i}^{2} \geq 0+\frac{1}{n-1}\left(\sum_{i \neq 1} b_{i, i}\right)^{2}
$$

by the Cauchy-Schwartz inequality and the fact that $b_{1,1}=0$. So,

$$
\mid \text { Hess }\left.b\right|^{2} \geq \frac{1}{n-1}\left((1 / 2-(v))(n-1) b^{-1}\right)^{2}=(n-1) b^{-2}(1 / 2-v)^{2} .
$$

Thus the inequalities must be equalities in the Cauchy-Schwartz inequality, (1.54), so

$$
b_{i, j}=0 \forall i, j \text { and } b_{k, k}=b_{l, l} \forall k, l \neq 1 .
$$

Using the formula for the Laplacian of $b$ once again we get

$$
b_{i, i}=(1 / 2-v) / b,
$$

and so we can solve for the warping function, $f(b)$,

$$
f^{\prime \prime}(b)=\frac{(1 / 2-v) f(b)}{b} \Longrightarrow f(b)=\left(\frac{b}{r_{0}}\right)^{\left(\frac{1}{2}-v\right)} f\left(r_{0}\right) \text {. }
$$


Thus $b^{-1}\left(\left(r_{1}, \infty\right)\right)$ is the desired warped product.

To complete the proof, note that the boundary, $b^{-1}\left(r_{1}\right)$, must be isometric to a rescaled $b^{-1}(r)$ for any $r>r_{1}$. So it is smooth and can be included in the warped product.

This completes the proof of Theorem 9 .

Note that if we only had the condition of minimal volume growth, then we could prove a series of lemmas similar to the ones proven here to show that $\Delta \bar{b}$ is approximately equal to $(n-1)(1 / 2-v) / \bar{b}$ in a weak sense. We could then apply Cheeger and Colding's Almost Rigidity Theory to prove the Theorem 33. Rather than imitating their methods from scratch, we will adapt one of their key theorems to our situation. However, the reader should understand that it is the control on the weak Laplacian of the Busemann function that gives us the almost rigidity.

\section{Minimal Volume Growth and Almost Rigidity.}

In this section, we examine the asymptotic properties of a manifold with a quadratically decaying lower Ricci curvature bound, (0.1), and minimal volume growth. [Recall Definition 2]. We wish to show that compact regions in such a manifold are Gromov-Hausdorff close to warped product manifolds [Theorem 33]. To do so, we will apply the following theorem proven in [So2].

Theorem 22 (So2, Thm 19). Let $M$ be a manifold with nonnegative Ricci curvature, a quadratically decaying lower Ricci curvature bound, (0.1) with $v \in[0,1 / 2]$, and minimal volume growth. Then the Busemann function, $b(x)$, has compact level sets and their diameter grows at most linearly,

$$
\operatorname{diam}\left(b^{-1}(r)\right) \leq C_{D}|r+1| \quad \forall r \geq r_{0}
$$

In order to apply this theorem, we will assume that all our manifolds have globally nonnegative Ricci curvature for the remainder of the paper. Thus the regions $b^{-1}([r, r+L])$ are compact. These are the compact regions which are proven to be close to warped product manifolds in the GromovHausdorff sense in Theorem 33.

The precise statement of the almost rigidity theorems, Theorems 33 and 34 will appear in 2.2 after the Gromov-Hausdorff distance and related. concepts are defined. 
Before going on, it is important to note the following facts from [So2, Cor 23] reviewed in Section 1.1. The function,

$$
V(r)=\frac{V o l_{n-1}\left(b^{-1}(r)\right)}{r^{(1 / 2-v)(n-1)}}
$$

is nondecreasing as a function of $r$ in any manifold with a quadratically decaying lower Ricci curvature bound, and, if the manifold also has nonnegative Ricci curvature and minimal volume growth, then

$$
\lim _{r \rightarrow \infty} \frac{V o l_{n-1}\left(b^{-1}(r)\right)}{r^{(1 / 2-v)(n-1)}}=V_{\infty}<\infty .
$$

In fact $V_{\infty}=V_{0}$ of the minimal volume growth definition [Defn 2] unless the manifold splits isometrically, in which case $V_{\infty}=V_{0} / 2$.

Both the constants $C_{D}$ and $V_{\infty}$ will be refered to in the proofs of our almost rigidity theorem and our diameter growth estimate.

\subsection{Almost Rigidity and the Gromov-Hausdorff Metric.}

There are a number of equivalent definitions of the Gromov-Hausdorff metric on the space of metric spaces. Here we will use the Gromov-Hausdorff map to define this metric, since ultimately we will use both the Gromov-Hausdorff closeness and the particular Gromov-Hausdorff map to prove our diameter theorem. See $[\mathrm{GrLaPa}]$ for more details.

Definition 23. Given $\varepsilon>0$, the Gromov Hausdorff distance, $d_{G H}(X, Y)$ between two compact metric spaces, $X$ and $Y$, is less than $\varepsilon$ if there exists a Gromov-Hausdorff map, $F_{G H}: X \mapsto Y$ which is $\varepsilon$-almost onto,

$$
T_{\varepsilon}\left(F_{G H}(X)\right) \supset Y,
$$

and $\varepsilon$-almost distance preserving,

$$
\left|d_{Y}\left(F_{G H}\left(x_{1}\right), F_{G H}\left(x_{2}\right)\right)-d_{X}\left(x_{1}, x_{2}\right)\right|<\varepsilon .
$$

The Gromov-Hausdorff map need not be continuous.

Note that this definition is not quite symmetric. However, if there exists $F_{G H}: X \mapsto Y$ with the above properties then the map $\bar{F}_{G H}: Y \mapsto X$, such that $\bar{F}_{G H}(y)$ equal any $x \in X$ such that $d_{X}\left(y, F_{G H}(x)\right)<\varepsilon$, is $2 \varepsilon$-almost distance preserving and $2 \varepsilon$-almost onto. 
Definition 24. Given any subset, $U$, of a length space, $N$, we can define a localized distance function,

$$
d_{U}(x, y)=\inf \{L(c([0,1])): c(0)=x, c(1)=y, c([0,1]) \subset U\}
$$

In particular, if we chose any constants $\alpha$ and $\alpha^{\prime}$ such that $0<\alpha^{\prime}<\alpha<$ $(b-a) / 2$ then we can define

$$
d^{\alpha^{\prime}}(x, y)=d_{r^{-1}\left(a+\alpha^{\prime}, b-\alpha^{\prime}\right)}(x, y)
$$

as a distance function on $r^{-1}\left(a+\alpha^{\prime}, b-\alpha^{\prime}\right)$ and its restriction $d^{\alpha, \alpha^{\prime}}$ to the subset $r^{-1}(a+\alpha, b-\alpha)$. There is a discussion of these two functions in [ChCo, Section 3].

Note 25. A localized component of $r^{-1}((a+\alpha, b-\alpha))$ is a set of the form $U \cap r^{-1}((a+\alpha, b-\alpha))$ where $U$ is a connected component of $r^{-1}((a+\alpha, b-\alpha))$. Thus $d^{\alpha, \alpha^{\prime}}(x, y)$ is finite iff $x$ and $y$ are in the same localized component.

When we say that two spaces are Gromov-Hausdorff close each of which has more than one such component, then we have paired off all the localized components and shown that each pair is Gromov-Hausdorff close. In particular, there are the same number of localized components [ChCo]. Note that in a warped product manifold localized components are connected components.

We now present a particular theorem of Cheeger and Colding which is especially useful in the study of manifolds with minimal volume growth. This theorem states that manifolds with lower Ricci curvature bounds and almost maximal volume with respect to a distance function, $r$, are GromovHausdroff close to certain warped product manifolds [ChCo, Thm 4.85]. First we provide a definition of almost maximal volume.

Let $m_{x}\left(r^{-1}(a)\right)$ denote the mean curvature of $r^{-1}(a)$ at the point $x$. We will omit the subscript $x$ when it is unimportant.

Definition 26. Let $N^{n}$ be a Riemannian manifold and $K \subset N^{n}$ compact. Let $r(x)=d(x, K)$ be the distance function to $K$. Fix $b>a \geq 0$.

If the region $r^{-1}(a, b) \subset N^{n}$ has the following three properties for some 
positive smooth function, $f$ and some $\omega \geq 0$

$$
\begin{gathered}
\operatorname{Ric}_{N^{n}}(x) \geq-(n-1) \frac{f^{\prime \prime}(r(x))}{f(r(x))} \\
m\left(r^{-1}(a)\right) \leq(n-1) \frac{f^{\prime}(a)}{f(a)} \text { on } r^{-1}(a) \\
\frac{V o l\left(r^{-1}(a, b)\right)}{V o l_{n-1}\left(r^{-1}(a)\right)} \geq(1-\omega) \frac{\int_{a}^{b} f^{n-1}(r) d r}{f^{n-1}(a)}
\end{gathered}
$$

then we say that the region $r^{-1}(a, b)$ has $\omega$-almost maximal volume with respect to the function $f$.

Theorem 27 (Cheeger and Colding). [ChCo, Thm 4.85]

Let $N^{n}$ have Ricci $\geq \Lambda$. Let $K \subset N^{n}$ be compact and let $r(x)=d(x, K)$. Let $f$ be a smooth nonnegative function.

Suppose a region $r^{-1}((a, b)) \subset N^{n}$ has $\omega$-almost maximal volume with respect to $f$.

Then there exists a bound,

$$
\Psi(\omega)=\Psi\left(\omega \mid n, f, a, b, \alpha, \alpha^{\prime}, \xi, \Lambda, \operatorname{diam}\left(r^{-1}(a, b)\right)\right.
$$

such that

$$
\lim _{\omega \rightarrow 0} \Psi\left(\omega \mid n, f, a, b, \alpha, \alpha^{\prime}, \xi, \Lambda, \operatorname{diam}\left(r^{-1}(a, b)\right)=0 .\right.
$$

and there exists a length space, $X$, such that

$$
d_{G H}\left(r^{-1}((a+\alpha, b-\alpha)), X \times_{f}(a+\alpha, b-\alpha)\right) \leq \Psi(\omega)
$$

where the region $r^{-1}((a, b))$ is endowed with the localized distance functions, $d^{\alpha, \alpha^{\prime}}$.

Note 28. The length space, $X$, defined by Cheeger and Colding is a length space defined to be arbitrarily close to the set, $r^{-1}\left(a+\alpha^{\prime}\right)$, endowed with a localized distance function $d_{U}$ with

$$
U=r^{-1}\left(a+\alpha^{\prime}-\xi, a+\alpha^{\prime}-\xi\right) .
$$

See [ChCo, Prop 3.3]. 
Note 29. More important for our purposes is the fact that the GromovHausdorff map for this theorem is defined,

$$
F_{G H}(x)=(\pi(x), r(x)) .
$$

Here $\pi(x)=f_{X}(\bar{\pi}(x))$, where $f_{X}: r^{-1}\left(a+\alpha^{\prime}\right) \mapsto X$ is a Gromov-Hausdorff map and $\bar{\pi}(x)$ is any point in $r^{-1}\left(\alpha+\alpha^{\prime}\right)$ closest to $x$ [ChCo Thm 3.6]. Note that $F_{G H}$ is not a uniquely determined function, nor is in continuous.

Note 30. In the process of proving that $F_{G H}$ is almost onto, see Lemma 3.38 of [ChCo], Cheeger and Colding prove a formula which implies that for any $t \in(a+\alpha, b-\alpha)$, the restricted function,

$$
F_{G H}: r^{-1}(t) \longmapsto X^{\prime} \times_{f}\{t\}
$$

is almost onto. (See [So1, Section 4.2] for more details). Thus $X^{\prime}$ and $X$ can be best described as being Gromov-Hausdorff close to any given level set $r^{-1}(t)$ rescaled by $f(t)$ with the localized distance function $d_{U}$ of Theorem 27.

The fact that $F_{G H}$ is a Gromov-Hausdorff map between level sets will be crucial to our proof of Theorem 45 .

Note 31. The estimating function

$$
\Psi\left(\omega \mid n, f, a, b, \alpha, \alpha^{\prime}, \xi, \Lambda, \operatorname{diam}\left(r^{-1}(a, b)\right)\right.
$$

of the Cheeger-Colding Theorem depends on the warping function, $f$, only through the following quantities:

$$
\begin{array}{ll}
K_{1} \geq \sup _{r \in[a, b]}|f(r)| & K_{3} \geq \sup _{r \in[a, b]}\left|f^{\prime}(r)\right| \\
K_{2} \geq \sup _{r \in[a, b]}\left|\frac{1}{f(r)}\right| & K_{4} \geq \sup _{r \in[a, b]}\left|\frac{f^{\prime \prime}(r)}{f(r)}\right|
\end{array}
$$

Note that we normalize $f(r)$ so that $f(a)=1$. See [So1, Sections 4.1-4.2] for details.

Remark 32. Cheeger and Colding do not state this theorem exactly as we have written it above. In their statement, the functions $\Psi, N$ and $D$ do not depend on $\operatorname{diam}\left(r^{-1}(a, b)\right)$ but instead on a function $\mathcal{V}$.

$$
\mathcal{V}(u)=\inf _{q \in r^{-1}(a, b)} \frac{\operatorname{Vol}\left(B_{u}(q)\right)}{\operatorname{Vol}\left(r^{-1}(a, b)\right)}
$$


By examining Prop. 4.50 and Lemmas 3.28 and 3.32 of [ChCo], where the dependence on $\mathcal{V}$ is introduced, and Prop 2.24 of [ChCo], which describes the properties of this function, it is clear that this dependence can be replaced by dependence on the minimum Ricci curvature, $\Lambda$, the dimension, $n$, and the diameter of the set measured with respect to the standard metric, $\operatorname{diam}\left(r^{-1}(a+\alpha, b-\alpha)\right.$. The restatement in Theorem 27 is convenient for our purposes.

\subsection{The Asymptotic Almost Rigidity of Manifolds with Minimal Volume Growth.}

We can now state our asymptotic almost rigidity theorem. Recall the Definition 2 of minimal volume growth. Recall the constant $V_{\infty}$ of (2.3).

Theorem 33. Let $M^{n}$ be a manifold with a ray $\gamma$, nonnegative Ricci curvature everywhere,

$$
\operatorname{Ricci}(x) \geq \frac{(n-1)\left(\frac{1}{4}-v^{2}\right)}{b(x)^{2}} \text { on } b^{-1}\left(\left[r_{0}, \infty\right)\right)
$$

where $v \in(0,1 / 2]$ and minimal volume growth.

Then for any given $\varepsilon>0$ and $L>\varepsilon>0$, there exists a sufficiantly large constant, $V_{\varepsilon, L}<V_{\infty}$, such that if

$$
\operatorname{Vol}_{(n-1)}\left(b^{-1}\left(r_{1}\right)\right) \geq V_{\varepsilon, L}\left(r_{1}\right)^{(n-1)(1 / 2-v)}
$$

then there exists a length space $X_{r_{1}}$ such that

$$
\begin{aligned}
d_{G H}\left(b^{-1}\left(\left(r_{1}+\varepsilon, r_{1}+L\right)\right), X_{r_{1}+\varepsilon} \times_{\left(b^{(1 / 2-v)}\right)}\left(r_{1}+\varepsilon,\right.\right. & \left.\left.r_{1}+L\right)\right)< \\
& <\varepsilon \operatorname{diam}\left(b^{-1}\left(r_{1}\right)\right) .
\end{aligned}
$$

This Gromov-Hausdorff closeness is from level set to level set [Note 30], so in fact $X_{r_{1}}$ is Gromov-Hausdorff close to any level $b^{-1}(s)$ rescaled by the warping function $f(s)$ as long as $s \in\left(r_{1}+\varepsilon, r_{1}+L\right)$.

The distance function on $b^{-1}\left(\left(r_{1}+\varepsilon, r_{1}+L\right)\right)$ and $b^{-1}(s)$ is the localized distance function, $d_{b^{-1}\left(r_{1}+\varepsilon / 2, r_{1}+L+\varepsilon / 2\right)}$.

The following is a corollary of the above or can be proven directly with a simplification of the above theorem's proof. 
Theorem 34. Given a manifold, $M^{n}$, with nonnegative Ricci curvature and linear volume growth, for any given $\varepsilon>0$ and $L>\varepsilon>0$, there exists a sufficiantly large constant,

$$
V_{\varepsilon, L}<\lim _{R \rightarrow \infty} \operatorname{Vol}_{n-1}\left(b^{-1}(R)\right)=V_{\infty}
$$

such that if

$$
\operatorname{Vol}_{(n-1)}\left(b^{-1}\left(r_{1}\right)\right) \geq V_{\varepsilon, L}>0
$$

then

$$
d_{G H}\left(b^{-1}\left(\left(r_{1}+\varepsilon, r_{1}+L\right)\right), X_{r_{1}} \times\left(r_{1}+\varepsilon, r_{1}+L\right)\right)<\varepsilon \operatorname{diam}\left(b^{-1}\left(r_{1}\right)\right) .
$$

Here $X_{r_{1}}$ is a length space such that

$$
d_{G H}\left(X_{r_{1}}, b^{-1}\left(r_{1}+\varepsilon\right)\right)<\varepsilon \operatorname{diam}\left(b^{-1}\left(r_{1}\right)\right) .
$$

All spaces in this theorem are endowed with the localized distance function $d_{U}$ with $U=b^{-1}\left(r_{1}+\varepsilon / 2, r_{1}+L+\varepsilon / 2\right)$.

Theorem 34 essentially asserts that once a level set has a large enough $(n-1)$-volume, then the nearby region is almost an isometric product of that level with an interval. Note that we are forced to shift our region over slightly in order to be able to match the components of the region to that of the level. Cheeger and Colding are only able to control the distances of a subregion of the original region because the estimates on the Hessian of the distance function are only controlled on subregions of the region where the volume is controlled. For this reason, all the distance functions are also localized inside subsets of the original region.

Note that these manifolds do not necessarily converge to unique warped product manifolds even if the diameter of the Busemann level sets is uniformly bounded. In [So2], there are examples of manifolds satisfying the hypothesis of Theorem 33 for which there exist $r_{i} \rightarrow \infty$ such that

$$
b^{-1}\left(\left(r_{2 i}, r_{2 i}+L\right)\right) \rightarrow X \times(0, L),
$$

and

$$
b^{-1}\left(\left(r_{2 i+1}, r_{2 i+1}+L\right)\right) \rightarrow Y \times(0, L),
$$

where $X$ and $Y$ are not isometric. In order to force the manifold to be asymptotically close to a unique isometric product manifold we would have to add additional conditions on the speed at which $V o l_{n-1}\left(b^{-1}(r)\right)$ approaches $V_{\infty}$. See Remarks 42 and 43 after the proof of Theorem 33. 


\subsection{Minimal Volume Growth and Almost Maximality.}

In this section we begin a proof of both Theorem 33 on asymptotic almost rigidity and Theorem 45 on the diameter growth of manifolds with minimal volume growth and globally nonnegative Ricci curvature. The key ingredient in both proofs is the application of the Cheeger Colding Theorem 27. Here we provide a series of lemmas which relate our hypothesis of minimal volume growth to their hypothesis of almost maximality. Recall Definitions 2 and 26.

In order to study noncompact manifolds with minimal volume growth, we do not examine standard distance functions, but instead we examine Busemann functions. The following lemma asserts that Busemann functions are distance functions on certain regions (See [So1] for the proof).

Lemma 35. Let $r_{2}$ be a real number. Let $r(x)=d\left(x, b^{-1}\left(r_{2}\right)\right)$. Then $r(x)=$ $r_{2}-b(x)$ on the region $b^{-1}\left(-\infty, r_{2}\right]$.

Recall that Theorem 22 states that the Busemann level sets are compact on manifolds with minimal volume growth and globally nonnegative Ricci curvature. Thus $r(x)=d\left(x, b^{-1}\left(r_{2}\right)\right)$ of the above lemma can be used as our distance function in the Cheeger-Colding Theorem [ChCo, 4.85].

Cheeger and Colding showed that regions with almost maximal volume were almost warped products. Here, we are studying compact regions in noncompact manifolds with minimal volume growth. These ideas are related because in a manifold with minimal volume growth, annuli about increasingly distant points, $\gamma\left(R_{i}\right)$, have almost maximal volume. Such annuli converge to regions between level sets of the Busemann function, $b_{\gamma}$. See Lemma 14 and [So2].

In the next lemma we show that once a Busemann level set in such noncompact manifold has sufficiently large (n-1)-volume, then any region beyond that level is $\omega$-almost maximal. Recall, also the definition of the $\omega$-almost maximal volume property in Definition 26 and of $V_{\infty}$ in (2.3).

Lemma 36. Let $M^{n}$ be a manifold with a ray $\gamma$ and Ricci curvature bounded below as in (0.1) with $v \in[0,(n+1) / 2(n-1))$. Suppose $M^{n}$ has minimal volume growth and that the level sets of the Busemann function are compact.

If $r_{1}$ is large enough that

$$
0<V_{\infty}-\frac{V o l_{n-1}\left(b^{-1}\left(r_{1}\right)\right)}{r_{1}^{p}}<\omega V_{\infty}
$$


then for all $r_{2}>r_{1}$, the region, $b^{-1}\left(\left(r_{1}, r_{2}\right)\right)=r^{-1}\left(0, r_{2}-r_{1}\right)$, where $r(x)=$ $r_{2}-b(x)=d\left(x, b^{-1}\left(r_{2}\right)\right)$, has the $\omega$-almost maximal volume property with respect to the function $f(r)=\left(r_{2}-r\right)^{\left(\frac{1}{2}-v\right)}$.

Proof. First, it is easy to check that $f(r)$ is the appropriate function for the Ricci bound because

$$
\begin{aligned}
-(n-1) \frac{f^{\prime \prime}(r(x))}{f(r(x))} & =-(n-1)\left(\frac{1}{2}-v\right)\left(-\frac{1}{2}-v\right) \frac{(-1)^{2}}{\left(r_{2}-r\right)^{2}}= \\
& =(n-1) \frac{\left(\frac{1}{4}-v^{2}\right)}{b^{2}(x)} .
\end{aligned}
$$

As for the mean curvature bound we know

$$
(n-1) \frac{f^{\prime}(0)}{f(0)}=(n-1) \frac{-\left(\frac{1}{2}-v\right)\left(r_{2}\right)^{\left(-\frac{1}{2}-v\right)}}{\left(r_{2}\right)^{\left(\frac{1}{2}-v\right)}}=\frac{-(n-1)\left(\frac{1}{2}-v\right)}{r_{2}}
$$

Using the Laplacian Comparison Theorem [Ch] and (1.7) which tells us the Laplacian of the Busemann function on our comparison warped product manifold, we get,

$$
\frac{-(n-1)\left(\frac{1}{2}-v\right)}{r_{2}} \geq-\Delta b=-m\left(b^{-1}\left(r_{2}\right)\right)=m\left(r^{-1}(0)\right) .
$$

Thus we have verified the mean curvature requirement.

So now we need only show

$$
\frac{V o l\left(r^{-1}(0, b)\right)}{V o l_{n-1}\left(r^{-1}(0)\right)} \geq(1-\omega) \frac{\int_{0}^{b} f^{n-1}(r) d r}{f^{n-1}(0)},
$$

or equivalently,

$$
\frac{\operatorname{Vol}\left(b^{-1}\left(r_{1}, r_{2}\right)\right)}{\operatorname{Vol}_{n-1}\left(b^{-1}\left(r_{2}\right)\right)} \geq(1-\omega) \frac{\int_{r_{1}}^{r_{2}} b^{(n-1)\left(\frac{1}{2}-v\right)} d b}{\left(r_{2}\right)^{(n-1)\left(\frac{1}{2}-v\right)}} .
$$

By Theorem 5 we have

$$
\begin{aligned}
\frac{V o l\left(b^{-1}\left(r_{1}, r_{2}\right)\right)}{V o l_{n-1}\left(b^{-1}\left(r_{2}\right)\right)} & =\frac{\int_{r_{1}}^{r_{2}} \operatorname{Vol}_{n-1}\left(b^{-1}(s)\right) d s}{V o l_{n-1}\left(b^{-1}\left(r_{2}\right)\right)} \\
& \geq \frac{\left(\int_{r_{1}}^{r_{2}}\left(\frac{s}{r_{1}}\right)^{(n-1)\left(\frac{1}{2}-v\right)} d s\right) \operatorname{Vol}_{n-1}\left(b^{-1}\left(r_{1}\right)\right)}{\operatorname{Vol}_{n-1}\left(b^{-1}\left(r_{2}\right)\right)} \\
& =\frac{V o l_{n-1}\left(b^{-1}\left(r_{1}\right)\right)}{V o l_{n-1}\left(b^{-1}\left(r_{2}\right)\right)} \frac{\int_{r_{1}}^{r_{2}} b^{(n-1)\left(\frac{1}{2}-v\right)} d b}{\left(r_{2}\right)^{(n-1)\left(\frac{1}{2}-v\right)}}\left(\frac{r_{2}}{r_{1}}\right)^{(n-1)\left(\frac{1}{2}-v\right)}
\end{aligned}
$$


So we need only show

$$
\frac{\operatorname{Vol}_{n-1}\left(b^{-1}\left(r_{1}\right)\right)}{\operatorname{Vol}_{n-1}\left(b^{-1}\left(r_{2}\right)\right)}\left(\frac{r_{2}}{r_{1}}\right)^{(n-1)\left(\frac{1}{2}-v\right)} \geq(1-\omega) .
$$

Now we are given

$$
\frac{\left(V_{\infty}-\frac{V o l_{n-1}\left(b^{-1}\left(r_{1}\right)\right)}{r_{1}^{(n-1)\left(\frac{1}{2}-v\right)}}\right)}{V_{\infty}} \leq \omega
$$

Using the monotonicity,

$$
\frac{V o l_{n-1}\left(b^{-1}\left(r_{1}\right)\right)}{r_{1}^{(n-1)\left(\frac{1}{2}-v\right)}}<\frac{V o l_{n-1}\left(b^{-1}\left(r_{2}\right)\right)}{r_{2}^{(n-1)\left(\frac{1}{2}-v\right)}} \leq V_{\infty}
$$

we find

$$
\frac{\left(\frac{V o l_{n-1}\left(b^{-1}\left(r_{2}\right)\right)}{r_{2}^{(n-1)\left(\frac{1}{2}-v\right)}}-\frac{V o l_{n-1}\left(b^{-1}\left(r_{1}\right)\right)}{r_{1}^{(n-1)\left(\frac{1}{2}-v\right)}}\right)}{\left(\frac{V o l_{n-1}\left(b^{-1}\left(r_{2}\right)\right)}{r_{2}^{(n-1)\left(\frac{1}{2}-v\right)}}\right)} \leq \omega .
$$

Cancelling the terms involving $r_{2}$ and rearranging this equation, we obtain (2.29) and we are done.

In the Cheeger Colding Theorem 27, the given region has a fixed lower Ricci curvature bound, diameter bound and comparison warping function. It is shown that the region is almost a warped product if its almost maximal volume estimate, $\omega$, is sufficiently close to 0 . The Gromov-Hausdorff closeness depends on

$$
\Psi\left(\omega_{\varepsilon, L} \mid n, f, a, b, \alpha, \alpha^{\prime}, \xi, \Lambda, \operatorname{diam}\left(r^{-1}(a, b)\right)\right)
$$

and $\Psi$ only approaches 0 when all the other parameters are fixed.

In our situation, we are examining the asymptotic behavior of a sequence of regions contained between Busemann levels, $b^{-1}\left(r_{1}, r_{2}\right)$, where $r_{1}$ and $r_{2}$ approach infinity. Thus our set $K=b^{-1}\left(r_{2}\right)$ will not be a fixed set and the diameters of the regions, $b^{-1}\left(r_{1}, r_{2}\right)$, will be changing. Thus we must rescale the regions before applying the Cheeger-Colding Theorem. 
Lemma 37. Let $N_{1}^{n}$ be a Riemannian manifold with a region $r_{1}^{-1}\left(a_{1}, b_{1}\right)$ that has $\omega$-almost maximal volume with respect to a function $f_{1}$. Let $N_{2}^{n}$ be the manifold $N_{1}^{n}$ with its metric scaled down by $D^{2}$. Then $r_{2}(x)=$ $\left(r_{1}(x)-a_{1}\right) / D$ is a distance function on the region $r_{2}^{-1}\left(0,\left(b_{1}-a_{1}\right) / D\right)$ in $N_{2}^{n}$. Furthermore, if we let $f_{2}(t)=f_{1}\left(t D+a_{1}\right) / D$ then the region, $r_{2}^{-1}\left(\left(0, \frac{b_{1}-a_{1}}{D}\right)\right)$, has $\omega$-almost maximal volume with respect to this function $f_{2}$.

The details of this proof can be found in [So1, Lemma 4.10].

Clearly, the rescaling of the manifold will affect other parameters in the Cheeger-Colding theorem. In particular, the distance between levels sets, $r^{-1}(a)$ and $r^{-1}(b)$ may become very small. This is a problem because the Cheeger-Colding Theorem requires that there be fixed constants $b-a>\alpha>$ $\alpha^{\prime}>0$. While we cannot employ the Cheeger-Colding Theorem to prove the Gromov-Hausdorff closeness in this situation it is easy to see that a thin set must be close to a warped product regardless of its volume properties. See [So1, Lemma 4.11] for details.

Lemma 38. Given a manifold, $M^{n}$, a compact subset, $K$, and a distance function $r(x)=d(x, K)$. Given any $\varepsilon>0$, if

$$
b-a<\delta_{\varepsilon}=\frac{\varepsilon}{4}
$$

then

$$
d_{G H}\left(r^{-1}(a, b), r^{-1}(\dot{a}) \times_{f}(a, b)\right)<\varepsilon .
$$

where the distance function on $r^{-1}(a, b)$ and $r^{-1}(a)$ can be any localized distance function, $d_{U}$, where $U \supset r^{-1}(a, b)$. (See Defn 24).

Before rescaling regions between level sets, we would like to estimate their diameter. Three different estimates are obtained in the following lemmas. The first lemma is simple but is used to prove both Theorem 33 and Theorem 45.

Lemma 39. Let $M^{n}$ be any complete noncompact Riemannian manifold with a Busemann function, $b$. Then, for all $r_{1}<r_{2}$,

$$
\operatorname{diam}\left(b^{-1}\left(r_{1}, r_{2}\right)\right) \leq \operatorname{diam}\left(b^{-1}\left(r_{2}\right)\right)+2\left(r_{2}-r_{1}\right) .
$$

Proof. Given any $x$ in $b^{-1}\left(r_{1}\right)$, there exists a Busemann ray, $\gamma_{x}$, which is parametrized by arclength, such that $\gamma_{x}\left(r_{1}\right)=x$ and $\gamma_{x}\left(r_{2}\right) \in b^{-1}\left(r_{2}\right)$. 
Thus to control the diameter of a region we need only control the diameter of the boundary closer to infinity.

The next lemma gives an explicit bound on the diameter of the boundary as a function of the diameter and volume of the first level set. This lemma cannot employ the Busemann rays to travel between the levels and thus requires minimal volume growth and globally nonnegative Ricci curvature. The techniques used to prove this lemma were developed in [So2].

Lemma 40. Let $M^{n}$ be a manifold with Ricci $\geq 0$ everywhere. Suppose it has Ricci Curvature bounded as in (0.1) with $v \in[0,1 / 4]$ and minimal volume growth.

Given any $0<\varepsilon<1 / 2$, we can find a level set with a sufficiently large volume

$$
\frac{V o l_{n-1}\left(b^{-1}\left(r_{1}\right)\right)}{p r_{1}^{p-1}}>V_{\infty}\left(1-\frac{1}{2}\left(\frac{\varepsilon}{2+2 \varepsilon}\right)^{n}\right)
$$

such for any $L>0$ and any $r_{3}>r_{1}$ the level sets $b^{-1}\left(r_{3}\right)$ and $b^{-1}\left(r_{3}+L\right)$ are Hausdorff close as subsets of $M^{n}$

$$
d_{H}\left(b^{-1}\left(r_{3}\right), b^{-1}\left(r_{3}+L\right)\right)<\varepsilon \operatorname{diam}\left(b^{-1}\left(r_{3}+L\right)\right)+L
$$

and the difference between their diameters is controlled

$$
\left|\operatorname{diam}\left(b^{-1}\left(r_{3}\right)\right)-\operatorname{diam}\left(b^{-1}\left(r_{3}+L\right)\right)\right|<\varepsilon \operatorname{diam}\left(b^{-1}\left(r_{3}+L\right)\right)+2 L .
$$

Proof. By the last lemma, we know that

$$
b^{-1}\left(r_{3}\right) \subset T_{\delta}\left(b^{-1}\left(r_{3}+L\right)\right) .
$$

Thus the diameter of the first level set can be no larger than that of the second level set plus $2 L$,

$$
\operatorname{diam}\left(b^{-1}\left(r_{3}\right)\right)<\operatorname{diam}\left(b^{-1}\left(r_{3}+L\right)\right)+2 L .
$$

So now we must bound $\operatorname{diam}\left(b^{-1}\left(r_{3}+L\right)\right)$ from above and show that it is contained in the appropriate tubular neighborhood of $b^{-1}\left(r_{3}\right)$. We will do this proof by contradiction.

Let $D=\operatorname{diam}\left(b^{-1}\left(r_{3}+L\right)\right)$. Suppose that

$$
b^{-1}\left(r_{3}+L\right) \not \subset T_{(\varepsilon D / 2)}\left(\Omega_{r_{3}, r_{3}+L+\varepsilon D / 2}\left(b^{-1}\left(r_{3}\right)\right)\right) \text {. }
$$


Then there is a point $x \in b^{-1}\left(r_{3}+L\right)$ such that

$$
\left.B_{x}(\varepsilon D / 2) \cap \Omega_{r_{3}, r_{3}+L+\varepsilon D / 2}\left(b^{-1}\left(r_{3}\right)\right)\right)=0 .
$$

Thus, by Theorem 5 , we have

$$
\begin{aligned}
& \operatorname{Vol}\left(B_{x}(\varepsilon D / 2)\right)< \\
& \quad<\operatorname{Vol}\left(b^{-1}\left(r_{3}+L-\varepsilon D / 2, r_{3}+L+\varepsilon D / 2\right)\right) \\
& \quad \quad-\operatorname{Vol}\left(\Omega_{r_{3}+L-\varepsilon D / 2, r_{3}+L+\varepsilon D / 2}\left(b^{-1}\left(r_{3}\right)\right)\right) \\
& \quad<\left(\left(r_{3}+L+\frac{\varepsilon D}{R}\right)^{p}-\left(r_{3}+L-\frac{\varepsilon D}{R}\right)^{p}\right)\left(V_{\infty}-\frac{V o l\left(b^{-1}\left(r_{3}\right)\right)}{p r_{3}^{p-1}}\right)
\end{aligned}
$$

On the other hand, by the Relative Volume Comparison Theorem [Bi] [Gr$\mathrm{LaPa}]$ and Ricci $\geq 0$ everywhere, we know

$$
\operatorname{Vol}\left(B_{x}(\varepsilon D / 2)\right)>\operatorname{Vol}\left(B_{x}(R)\right)\left(\frac{\varepsilon D / 2}{R}\right)^{n}
$$

We set $R=\operatorname{diam}\left(b^{-1}\left(r_{3}+L\right)\right)+2(\varepsilon D / 2)=D+2(\varepsilon D / 2)$ to insure that the ball of radius $R$ contains $b^{-1}\left(r_{3}+L-\varepsilon D / 2, r_{3}+L\right)$. Thus

$$
\begin{gathered}
\operatorname{Vol}\left(B_{x}(\varepsilon D / 2)\right)>\operatorname{Vol}\left(b^{-1}\left(r_{3}+L-\varepsilon D / 2, r_{3}+L\right)\right)\left(\frac{\varepsilon D / 2}{R}\right)^{n} \\
>\frac{V o l_{n-1}\left(b^{-1}\left(r_{3}\right)\right)}{p r_{3}^{p-1}}\left(\left(r_{3}+L+\frac{\varepsilon D}{R}\right)^{p}-\left(r_{3}+L-\frac{\varepsilon D}{R}\right)^{p}\right) . \\
\cdot\left(\frac{\varepsilon D / 2}{D+2(\varepsilon D / 2)}\right)^{n} .
\end{gathered}
$$

This last line employs Theorem 5 once again.

Using these two bounds for $\operatorname{Vol}\left(B_{x}(\varepsilon D / 2)\right)$, we have

$$
\begin{gathered}
\left(\left(r_{3}+L+\frac{\varepsilon D}{R}\right)^{p}-\left(r_{3}+L-\frac{\varepsilon D}{R}\right)^{p}\right)\left(V_{\infty}-\frac{V o l_{n-1}\left(b^{-1}\left(r_{3}\right)\right)}{p r_{3}^{p-1}}\right)> \\
>\frac{V o l_{n-1}\left(b^{-1}\left(r_{3}\right)\right)}{p r_{3}^{p-1}}\left(\left(r_{3}+L+\frac{\varepsilon D}{R}\right)^{p}-\left(r_{3}+L-\frac{\varepsilon D}{R}\right)^{p}\right) . \\
\cdot\left(\frac{\varepsilon D / 2}{D(1+\varepsilon)}\right)^{n}
\end{gathered}
$$


which we can rewrite as

$$
\frac{\left(V_{\infty} p r_{3}^{p-1}-V o l_{n-1}\left(b^{-1}\left(r_{3}\right)\right)\right)}{V o l_{n-1}\left(b^{-1}\left(r_{3}\right)\right)}>\left(\frac{\varepsilon / 2}{(1+\varepsilon)}\right)^{n} \frac{1}{2} .
$$

Now we take $r_{3}$ large enough for our volume estimate given by our choice of $r_{1}$ in (2.37) and get

$$
\begin{aligned}
\frac{1}{2}\left(\frac{\varepsilon}{2+2 \varepsilon}\right)^{n} & >\frac{V_{\infty} p r_{3}^{p-1}-V o l_{n-1}\left(b^{-1}\left(r_{1}\right)\right)}{V_{\infty}} \\
& >\frac{\left(V_{\infty} p r_{3}^{p-1}-V o l_{n-1}\left(b^{-1}\left(r_{3}\right)\right)\right)}{V o l_{n-1}\left(b^{-1}\left(r_{3}\right)\right)} \\
& >\left(\frac{\varepsilon / 2}{(1+\varepsilon)}\right)^{n} \frac{1}{2}
\end{aligned}
$$

which is a contradiction.

Thus our assumption in (2.41) does not hold and instead we have

$$
b^{-1}\left(r_{3}+L\right) \subset T_{(\varepsilon D / 2)}\left(\Omega_{r_{3}, r_{3}+L+\varepsilon D / 2}\left(b^{-1}\left(r_{3}\right)\right)\right) \text {. }
$$

Since all points in $\Omega_{r_{3}, r_{3}+L+\varepsilon D / 2}\left(b^{-1}\left(r_{3}\right)\right)$ are on segments of Busemann rays running from $b^{-1}\left(r_{3}\right)$ of length less than or equal to $L+\varepsilon D / 2$, we have

$$
\left.\Omega_{r_{3}, r_{3}+L+\varepsilon D / 2}\left(b^{-1}\left(r_{3}\right)\right)\right) \subset T_{(L+\varepsilon D / 2)}\left(b^{-1}\left(r_{3}\right)\right) .
$$

So, combining (2.48) and (2.49) we get

$$
b^{-1}\left(r_{3}+L\right) \subset T_{(L+\varepsilon D / 2+\varepsilon D / 2)}\left(b^{-1}\left(r_{3}\right)\right)
$$

where $D=\operatorname{diam}\left(b^{-1}\left(r_{3}+L\right)\right)$.

The lemma then follows.

We end this section with an easy rough estimate for a lower bound on the diameter of a Busemann level set as a function of its (n-1)-volume. Once again we restrict ourselves to manifolds with nonnegative Ricci curvature everywhere. However, we do not assume that we have minimal volume growth.

Lemma 41. In a complete noncompact manifold with nonnegative Ricci curvature such that $b^{-1}(r)$ is compact, we have

$$
\operatorname{diam}\left(b^{-1}(r)\right)>\left(\frac{V o l_{n-1}\left(b^{-1}(r)\right)}{w_{n} 2^{n}}\right)^{(1 /(n-1))}
$$


Proof. Let $d=\operatorname{diam}\left(b^{-1}(r)\right)$.

For any $x \in b^{-1}(r), B_{x}(2 d) \supset b^{-1}(r, r+d)$, so

$$
\operatorname{Vol}\left(B_{x}(2 d)\right)>\operatorname{Vol}\left(b^{-1}(r, r+d)\right)
$$

By the Bishop Volume Comparison Theorem [Bi, BiCr],

$$
w_{n}(2 d)^{n} \geq \operatorname{Vol}\left(B_{x}(2 d)\right) .
$$

Since $b$ is Lipschitz we can employ the Coarea formula [Fed 3.2.11] and Lemma 5 to get,

$$
\operatorname{Vol}\left(b^{-1}(r, r+d)\right) \geq(d) \operatorname{Vol}_{n-1}\left(b^{-1}(r)\right) .
$$

Thus

$$
w_{n}(2 d)^{n}>(d) \operatorname{Vol}_{n-1}\left(b^{-1}(r)\right)
$$

and

$$
d^{n-1}>\frac{V o l_{n-1}\left(b^{-1}(r)\right)}{w_{n} 2^{n}}
$$

\subsection{The Proof of the Asymptotic Almost Rigidity Theorem.}

We now prove Theorem 33. Throughout this section $M^{n}$ satisfies the hypotheses of this theorem. See Section 2.2.

We will begin by rescaling the region, $b^{-1}\left(r_{1}, r_{1}+L+\varepsilon\right)$, so that the diameter of the rescaled region is bounded above. Then we can apply the Cheeger Colding Theorem. Since,

$$
\operatorname{diam}\left(b^{-1}\left(r_{1}, r_{1}+L+\varepsilon\right) \leq 2(L+\varepsilon)+\operatorname{diam}\left(b^{-1}\left(r_{1}+\varepsilon\right)\right.\right.
$$

by Lemma 39 , we divide the metric by $D^{2}$ where

$$
D=\operatorname{diam}\left(b^{-1}\left(r_{1}+L+\varepsilon\right)\right) .
$$

We will first prove that given any $\varepsilon>0$ and any $L>\varepsilon>0$ there exists $V_{\varepsilon, L}$ sufficiently large that if

$$
\operatorname{Vol}_{(n-1)}\left(b^{-1}\left(r_{1}\right)\right) \geq V_{\varepsilon, L}\left(r_{1}\right)^{(n-1)(1 / 2-v)}
$$


then

$$
d_{G H}\left(b^{-1}\left(\left(r_{1}+\varepsilon, r_{1}+L\right)\right), X_{r_{1}+\varepsilon} \times_{\left(b^{(1 / 2-v)}\right)}\left(r_{1}+\varepsilon, r_{1}+L\right)\right)<\varepsilon^{\prime} D
$$

where

$$
\varepsilon^{\prime} \leq \frac{\varepsilon\left(\frac{V_{\infty}}{2^{n+1}}\right)^{1 /(n-1)}}{2\left(\frac{V_{\infty}}{2^{n+1}}\right)^{1 /(n-1)}+4 L+4 \varepsilon}
$$

This strange choice of $\varepsilon^{\prime}$ has been made so that later we can replace the dependence on diam $\left(b^{-1}\left(r_{1}+L+\varepsilon\right)\right)$ by $\operatorname{diam}\left(b^{-1}\left(r_{1}\right)\right)$ using Lemma 40.

We let $r(x)=d\left(x, b^{-1}\left(r_{1}+L+\varepsilon\right)\right)=\left(r_{1}+L+\varepsilon-b(x)\right) / D$ in this rescaled region. Note that $r(x)$ increases as $b(x)$ decreases and that this region is thus $r^{-1}(0, b)$ where

$$
b=\frac{L+\varepsilon}{D} .
$$

When $b<2 \varepsilon^{\prime}$, the region is "thin" and we know

$$
d_{G H}\left(r^{-1}(a, b), r^{-1}(a) \times_{f}(a, b)\right)<\varepsilon^{\prime} .
$$

for any warping function $f$ by Lemma 38. Thus (2.60) holds when we rescale back to the original region.

So we will assume $b \geq 2 \varepsilon$ and apply the Cheeger-Colding Theorem to obtain (2.63). First we must bound all the parameters in

$$
\Psi\left(\omega \mid n, f, a, b, \alpha, \alpha^{\prime}, \xi, \Lambda, \operatorname{diam}\left(r^{-1}(a, b)\right)\right)
$$

of the Cheeger-Colding theorem for our rescaled region. We set $n=n$, the dimension of our manifold. Since Ricci $\geq 0$ globally, we have $\Lambda=0$. We have $a=0$ and

$$
b=\frac{L+\varepsilon}{D} \leq \frac{L+\varepsilon}{\left(\frac{V o l_{n-1}\left(b^{-1}\left(r_{1}+L+\varepsilon\right)\right.}{2^{n}}\right)^{(1 /(n-1)}}
$$

by Lemma 41 which provides a lower bound on the diameter in terms of the volume. If we take

$$
V o l_{n-1}\left(b^{-1}\left(r_{1}+L+\varepsilon\right)\right) \geq V_{\varepsilon, L}>V_{\infty} / 2
$$


we can bound $b$ from above by a constant

$$
b \leq \frac{L+\varepsilon}{\left(\frac{V_{\infty} / 2}{2^{n}}\right)^{(1 /(n-1))}} .
$$

The other parameters can be set $\alpha^{\prime}=\alpha / 2$ and $\chi=\alpha^{\prime} / 2$ where

$$
\alpha=\frac{\varepsilon}{\operatorname{diam}\left(b^{-1}\left(r_{1}+L+\varepsilon\right)\right)}>\frac{b \varepsilon}{(L+\varepsilon)} .
$$

so they are all bounded from below and above as well.

The warping function, $f$, normalized such that $f(0)=1$, is

$$
f(r)=\left(\frac{r_{1}+D b-D r}{r_{1}+D b}\right)^{\left(\frac{1}{2}-v\right)} .
$$

To employ the Cheeger-Colding Theorem, we must bound this warping function as described in Remark 31. More specifically, to bound the constants, $K_{i}$, uniformly for all values of $r_{1}$ since we will have to vary $r_{1}$ to obtain the $\omega$-almost maximality required by the Cheeger-Colding Theorem. This is especially troublesome because $D=\operatorname{diam}\left(b^{-1}\left(r_{1}+L+\varepsilon\right)\right)$ depends on $r_{1}$ and may approach infinity.

To bound the constants $K_{i}$ which depend on $f$, we will use the fact that the diameter of the Busemann levels grows at most linearly [Theorem 22]. We must chose

$$
K_{1}=\sup _{r \in[0, b]}|f(r)|=\left(\frac{r_{1}+D b}{r_{1}+D b}\right)^{\left(\frac{1}{2}-v\right)}=1 .
$$

We need

$$
K_{2} \geq \sup _{r \in[0, b]}\left|\frac{1}{f(r)}\right|=\left(\frac{r_{1}}{r_{1}+D b}\right)^{-\left(\frac{1}{2}-v\right)} .
$$

Now $b$ is bounded above and below so we need only worry about $r_{1}$ and $D$ as $r_{1}$ approaches infinity. By the at most linear diameter growth, we have $D_{r_{1}} \leq C r_{1}$; so

$$
\lim _{r_{1} \rightarrow \infty}\left(\frac{r_{1}}{r_{1}+D b}\right)^{-\left(\frac{1}{2}-v\right)} \leq\left(\frac{1}{1+C b}\right)^{-\left(\frac{1}{2}-v\right)} .
$$


and $K_{2}$ exists. We must chose $K_{3}$ such that

$$
\begin{aligned}
K_{3} & \geq \sup _{r \in[0, b]}\left|f^{\prime}(r)\right| \\
& =\sup _{r \in[0, b]}\left|\left(\frac{1}{2}-v\right)\left(\frac{r_{1}+D b-D r}{r_{1}+D b}\right)^{\left(-\frac{1}{2}-v\right)}\left(\frac{-D}{r_{1}+D b}\right)\right| \\
& =\left(\frac{1}{2}-v\right)\left(\frac{D}{r_{1}+D b}\right)\left(\frac{r_{1}}{r_{1}+D b}\right)^{\left(-\frac{1}{2}-v\right)} .
\end{aligned}
$$

Once again we verify that the right hand side is bounded as $r_{1}$ goes to infinity,

$$
\begin{gathered}
\lim _{r_{1} \rightarrow \infty}\left(\frac{1}{2}-v\right)\left(\frac{D}{r_{1}+D b}\right)\left(\frac{r_{1}}{r_{1}+D b}\right)^{\left(-\frac{1}{2}-v\right)} \leq \\
\leq\left(\frac{1}{2}-v\right)\left(\frac{C}{1}\right)\left(\frac{1}{1+C b}\right)^{\left(-\frac{1}{2}-v\right)} .
\end{gathered}
$$

Finally we need to chose $K_{4}$ such that

$$
\begin{aligned}
K_{4} & \geq \sup _{r \in[0, b]}\left|\frac{f^{\prime \prime}(r)}{f(r)}\right| \\
& =\sup _{r \in[0, b]}\left(\frac{1}{2}-v\right)\left|-\frac{1}{2}-v\right|\left(\frac{D}{r_{1}+D b}\right)^{2}\left(\frac{r_{1}+D b-D r}{r_{1}+D b}\right)^{-2} \\
& =\left(\frac{1}{2}-v\right)\left(\frac{1}{2}+v\right)\left(\frac{D}{r_{1}+D b}\right)^{2}\left(\frac{r_{1}}{r_{1}+D b}\right)^{-2} .
\end{aligned}
$$

Once again we check if this is bounded as $r_{1}$ approaches infinity

$$
\begin{aligned}
\lim _{r_{1} \rightarrow \infty}\left(\frac{1}{2}-v\right)\left(\frac{1}{2}+v\right)\left(\frac{D}{r_{1}+D b}\right)^{2}\left(\frac{r_{1}}{r_{1}+D b}\right)^{-2} \leq \\
\leq\left(\frac{1}{2}-v\right)\left(\frac{1}{2}+v\right)\left(\frac{C}{1}\right)^{2}\left(\frac{1}{1+C b}\right)^{-2}
\end{aligned}
$$

Thus we have uniformly fixed our constants $K_{i}$.

For fixed $\varepsilon$ and $L$ and keeping all our parameters bounded as above, we can find an $\omega_{\varepsilon, L}$, depending only on $\varepsilon$ and $L$ such that

$$
\Psi\left(\omega_{\varepsilon, L} \mid n, f, a, b, \alpha, \alpha^{\prime}, \xi, \Lambda, \operatorname{diam}\left(r^{-1}(a, b)\right)\right)<\varepsilon^{\prime},
$$


where $\varepsilon^{\prime}$ is defined in (2.61).

If we can show that $r^{-1}(a, b)$ is $\omega_{\varepsilon, L}$-almost maximal, then by the Cheeger-Colding Theorem we have

$$
d_{G H}\left(r^{-1}(a+\alpha, b-\alpha), Y \times_{f}(a+\alpha, b-\alpha)\right)<\varepsilon^{\prime},
$$

where $Y$ is a length space and each level $b^{-1}(s)$ is in fact mapped by the Gromov-Hausdorff equivalence map almost onto $f(s) Y$ [Note 30]. The distance function on the metric product and the region are the localized distance functions $d^{\alpha, \alpha^{\prime}}=d_{r^{-1}\left(a+\alpha^{\prime}, b-\alpha^{\prime}\right)}$ defined in Definition 24 .

By the Lemma 37 we need only show that $b^{-1}\left(r_{1}, R_{1}+L+\varepsilon\right)$ has $\omega_{\varepsilon, L^{-}}$ almost maximal volume to show that the rescaled region $r^{-1}(a, b)$ is $\omega_{\varepsilon, L^{-}}$ almost maximal as well. By Lemma 36 , we know that if we take $r_{1}$ large enough that $\operatorname{Vol}_{n-1}\left(b^{-1}\left(r_{1}\right)\right) \geq V_{\varepsilon, L}$ where

$$
V_{\varepsilon, L}>\left(1-\omega_{\varepsilon, L}\right) V_{\infty}
$$

then $b^{-1}\left(r_{1}, R_{1}+L+\varepsilon\right)$ has $\omega_{\varepsilon, L}$-almost maximal volume. Thus (2.74) holds.

If we rescale the region $r^{-1}(a+\alpha, b-\alpha)$ back up to the original region $b^{-1}\left(r_{1}+\varepsilon, r_{1}+L\right)$, then we can rescale (2.74) to get

$$
d_{G H}\left(b^{-1}\left(\left(r_{1}+\varepsilon, r_{1}+L\right)\right), X_{r_{1}+\varepsilon} \times_{\left(b^{(1 / 2-v)}\right)}\left(r_{1}+\varepsilon, r_{1}+L\right)\right)<\varepsilon^{\prime} D,
$$

where the distance function on these spaces is the localized distance function, $d_{b^{-1}}\left(r_{1}+\varepsilon / 2, r_{1}+L+\varepsilon / 2\right)$. The distance function rescales in this manner because $\alpha^{\prime}=\alpha / 2=\varepsilon /(2 D)$. Since this closeness holds on each level set, $X_{r_{1}+\varepsilon}$ is close to $b^{-1}\left(r_{1}+\varepsilon\right)$.

Thus we have obtained (2.60). To complete the proof of our theorem we need to show that (2.76) holds if we replace the $\varepsilon^{\prime} \operatorname{diam}\left(b^{-1}\left(r_{1}+L+\varepsilon\right)\right)$ by $\operatorname{ediam}\left(b^{-1}\left(r_{1}\right)\right)$.

This may require us to take $r_{1}$ a little further out so that the volume of its level set is close enough to $V_{\infty}$ to employ Lemma 40 . That is, we take

$$
\frac{V o l_{n-1}\left(b^{-1}\left(r_{1}\right)\right)}{p r_{1}^{p-1}}>V_{\varepsilon, L}>V_{\infty}\left(1-\left(\frac{1}{6}\right)^{n}\right)>\frac{V_{\infty}}{2},
$$

which implies that

$$
V_{\infty}-\frac{V o l_{n-1}\left(b^{-1}\left(r_{1}\right)\right)}{p r_{1}^{p-1}}<V_{\infty}\left(\frac{1 / 2}{2+2(1 / 2)}\right)^{n}
$$


So we by Lemma 40, we know that

$$
\operatorname{diam}\left(b^{-1}\left(r_{1}+L+\varepsilon\right)\right)<2 \operatorname{diam}\left(b^{-1}\left(r_{1}\right)\right)+4(L+\varepsilon) .
$$

Using this information, we can rewrite our estimate in (2.60) as

$$
\begin{aligned}
d_{G H} & \left(b^{-1}\left(\left(r_{1}+\varepsilon, r_{1}+L\right)\right), X_{r_{1}+\varepsilon} \times_{b^{1 / 2-v}}\left(r_{1}+\varepsilon, r_{1}+L\right)\right)< \\
& <\frac{\varepsilon\left(\frac{V_{\infty} / 2}{w-n 2^{n}}\right)^{(1 /(n-1))}}{\left(2\left(\frac{V_{\infty} / 2}{w-n 2^{n}}\right)^{(1 /(n-1))}+4(L+\varepsilon)\right)} \operatorname{diam}\left(b^{-1}\left(r_{1}+L+\varepsilon\right)\right) \\
& <\frac{\varepsilon\left(\frac{V_{\infty} / 2}{w-n 2^{n}}\right)^{(1 /(n-1))}}{\left(2\left(\frac{V_{\infty} / 2}{w-n 2^{n}}\right)^{(1 /(n-1))}+4(L+\varepsilon)\right)}\left(2 \operatorname{diam}\left(b^{-1}\left(r_{1}\right)\right)+4(L+\varepsilon)\right) .
\end{aligned}
$$

By (2.77), we have $V o l_{n-1}\left(b^{-1}\left(r_{1}\right)\right)>V_{\infty} / 2$; so by Lemma 41 we know that

(2.80) $\operatorname{diam}\left(b^{-1}\left(r_{1}\right)\right)>\left(\frac{\operatorname{Vol}\left(b^{-1}\left(r_{1}\right)\right.}{w_{n} 2^{n}}\right)^{(1 /(n-1))}>\left(\frac{V_{\infty} / 2}{w-n 2^{n}}\right)^{(1 /(n-1))}$.

Since $D /(2 D+4(L+\varepsilon))$ is a decreasing function of $\mathrm{D}$, we can substitute this diameter estimate in the Gromov-Hausdorff estimate to get

$$
\begin{aligned}
d_{G H}\left(b^{-1}(\right. & \left.\left.r_{1}+\varepsilon, r_{1}+L\right), b^{-1}\left(r_{1}+\varepsilon\right) \times\left(r_{1}+\varepsilon, r_{1}+L\right)\right)< \\
& <\frac{\varepsilon \operatorname{diam}\left(b^{-1}\left(r_{1}\right)\right)}{\left(2 \operatorname{diam}\left(b^{-1}\left(r_{1}\right)\right)+4(L+\varepsilon)\right)}\left(2 \operatorname{diam}\left(b^{-1}\left(r_{3}\right)\right)+4(L+\varepsilon)\right) \\
& =\varepsilon \operatorname{diam}\left(b^{-1}\left(r_{1}\right)\right) .
\end{aligned}
$$

and we have completed the proof of Theorem 33 .

Note that in $V_{\varepsilon, L}$ was chosen in (2.75) and (2.77).

We could also consider manifolds in which the function,

$$
\delta(r):=V_{\infty}-\frac{V o l_{n-1}\left(b^{-1}(r)\right)}{r^{p-1}}
$$

decreases at given rate. This would give us results which are stronger than those implied by minimal volume growth but weaker than those implied by stongly minimal volume growth. 
Remark 42. If we assume that $\delta(r)$ decreases sufficiently fast, regions of increasing length, like $b^{-1}(r, 2 r)$, could be shown to be Gromov-Hausdorff close to warped product manifolds. This can be seen because we know that for any fixed set of parameters, we can choose $\delta(r)$ such that Gromov Hausdorff estimating function satisfies

$$
\Psi\left(\delta(r) \mid n, f, r, 2 r, \alpha, \alpha^{\prime}, \xi, 0, D r\right)<\varepsilon .
$$

Note that we are using at most linear diameter growth here to say that the diameter of $b^{-1}(r, 2 r)$ is less than $D r$, for some constant, $D$. Note also that we do not bother to rescale the manifold. Such a theorem would tell us that

$$
d_{G H}\left(b^{-1}(r, 2 r), b^{-1}(r) \times_{f}(r, 2 r)\right)<\varepsilon .
$$

In particular, we would truely see the warping of such a manifold.

Remark 43. If we were to take a function, $\delta(r)$, that decreased even faster, the manifold could be shown to be close to a unique warped product manifold. That is, if we choose $\delta(r)$ such that for all $r>r_{0}$ we have

$$
\Psi\left(\delta(r) \mid n, f, a=r, b=2 r, \alpha, \alpha^{\prime}, \xi, 0, D r\right)<\varepsilon\left(\frac{1}{2 r}\right),
$$

then

$$
d_{G H}\left(b^{-1}(r, 2 r), b^{-1}(r) \times_{f}(r, 2 r)\right)<\varepsilon\left(\frac{1}{2 r}\right) .
$$

Since our Gromov-Hausdorff map is from level set to level set, if we let $\frac{b^{-1}(r)}{f(r)}$ denote a level set with a localized metric rescaled by the warping function $f(r)$, then

$$
d_{G H}\left(\frac{b^{-1}(r)}{f(r)}, \frac{b^{-1}(2 r)}{f(2 r)}\right)<\varepsilon\left(\frac{1}{2 r}\right)
$$

Then, for all $k$,

$$
d_{G H}\left(\frac{b^{-1}\left(r_{0}\right)}{f\left(r_{0}\right)}, \frac{b^{-1}\left(2^{N} r_{0}\right)}{f\left(2^{N} r_{0}\right)}\right)<\varepsilon \sum_{k=1}^{N+1}\left(\frac{1}{2^{k} r_{0}}\right)<\frac{\varepsilon}{r_{0}} .
$$

Thus,

$$
d_{G H}\left(b^{-1}\left(r_{0}, \infty\right), b^{-1}\left(r_{0}\right) \times_{f}\left(r_{0}, \infty\right)\right)<\varepsilon
$$

and the manifold is close to a unique warped product manifold. 


\section{Linear Volume Growth and Sublinear Diameter Growth.}

In this section, we show that a manifold with nonnegative Ricci curvature everywhere and linear volume growth has sublinear diameter growth [Theorems 45 and 1]. We prove this both for diameters measured in the ambient manifold and for localized diameters as defined below in Definition 44.

In the previous section, we proved that in such a manifold, the region $b^{-1}((r, r+L))$ is almost an isometric product after rescaling by the diameter and taking $r$ large. Thus the "diameters" of $b^{-1}(r)$ and $b^{-1}(r+L)$ are close but only after rescaling by the diameter of $b^{-1}(r)$. To get sublinear diameter growth, we need to control increasingly long regions, $b^{-1}((r / 2,2 r))$ which allows us to compare $b^{-1}(r / 2)$ to $b^{-1}(2 r)$. Here we will rescale by dividing out by $r$ and we use the fact that $\operatorname{diam}\left(b^{-1}(r)\right) \leq C_{D} r$ where $C_{D}$ is the constant from Theorem 22, before applying Theorem 27 of [ChCo] and Lemmas 36, 37, and 38 from Section 2.3 .

Recall the definitions of localized distance and localized component from the Section 2.1 [Defn 24, [Note 25]] .

Throughout this chapter, "diameter" and diam, stated alone, refer to the diameter measured in the ambient manifold. We will now define the localized diameter or, more precisely, the $s$-almost intrinsic diameter.

Definition 44. Given any $s>0$, let $U=b^{-1}(R-s, R+s)$. The $s$-almost intrinsic diameter or localized diameter of a level set of a Busemann function is

$$
\left.\operatorname{diam}_{s}\left(b^{-1}(R)\right)=\max \left\{\operatorname{diam}_{V}\left(V \cap b^{-1}(R)\right)\right): V \text { is a conn comp of } U\right\},
$$

where

$$
\operatorname{diam}_{V}\left(V \cap b^{-1}(R)\right)=\sup _{x, y \in V \cap b^{-1}(R)} \inf _{\substack{c([0,1]) \subset V \\ c(0)=x, c(1)=y}} \operatorname{Length}(c) .
$$

This kind of diameter has been analyzed by Abresch and Gromoll [AbGl]. They proved that the almost intrinsic diameters of distance spheres in a manifold with nonnegative Ricci curvature grow linearly. This almost intrinsic diameter of the sphere of radius $R$ was defined to be the diameter of the largest component of the sphere measured with respect to a localized dis-

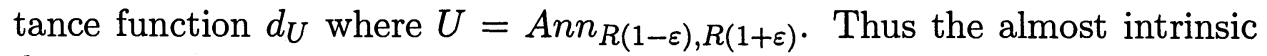
diameter was measured in terms of increasingly thick annuli. 
In Theorem 45 below, our almost intrinsic diameter of Busemann levels is also measured in this way. However, we prove that the diameter of the Busemann levels grows sublinearly and we assume that the manifold has linear volume growth.

Theorem 45. Let $M^{n}$ be a manifold with nonnegative Ricci curvature and linear volume growth. Then given any $\delta \in(0,1)$, we have sublinear almost intrinsic diameter growth,

$$
\lim _{R \rightarrow \infty} \frac{\operatorname{diam}_{\delta R}\left(b^{-1}(R)\right)}{R}=0 .
$$

After proving this theorem, we conclude the paper by showing that manifolds with linear volume growth and nonnegative Ricci curvature have sublinear diameter growth as well [Theorem 1]. This final theorem does not follow directly from the sublinear almost intrinsic diameter growth because of the lack of control on the number of localized components of the level sets.

Lemma 46. Given any $\psi>0$ there exists $R_{\psi}>0$ such that for all $r \geq R_{\psi}$, there exists a length space, $X_{r}$, such that

$$
d_{G H}\left(b^{-1}([r / 2,3 r]), X_{r} \times[r / 2,3 r]\right) \leq \psi r
$$

where the localized distance function on $b^{-1}([r / 2,3 r])$ is $d_{W}$ where

$$
W=b^{-1}((r / 2-\psi r / 2,3 r+\psi r / 2)) .
$$

Furthermore the Gromov Hausdorff Equivalence map from $b^{-1}([r / 2,3 r])$ to $X_{r} \times[r / 2,3 r]$ is $(\pi(x), b(x))$ where $\pi: b^{-1}(s) \mapsto X_{r}$ is a Gromov-Hausdorff equivalence map itself for all $s \in[r / 2,3 r]$ with respect to $d_{W}$.

Before proving this lemma, we make a few remarks pointing out some useful implications. Note that we are strongly using the fact that $X_{r} \times$ $[r / 2,3 r]$ is an isometric product.

Remark 47. Since $F_{G H}$ is almost onto, for all $(x, s) \in X_{r} \times[r / 2,3 r]$ there exists $x_{s} \in b^{-1}([r / 2,3 r]) \subset M^{n}$ such that $d_{X_{r} \times[r / 2,3 r]}\left(F_{G H}\left(x_{s}\right),(x, s)\right)<\psi r$. In particular, $d_{X_{r}}\left(\pi\left(x_{s}\right), x\right)<\psi r$ and $\left|b\left(x_{s}\right)-s\right|<\psi r$. Furthermore, for all $y_{1}, y_{2} \in b^{-1}([r / 2,3 r]), d_{X_{r}}\left(\pi\left(y_{1}\right), \pi\left(y_{2}\right)\right)<d_{W}\left(y_{1}, y_{2}\right)+\psi r$. 
Remark 48. In Lemma 46 we have implicitly stated that the region, $W=$ $b^{-1}((r / 2-\psi r, 3 r+\psi r))$, has the same number of connected components as $X_{r} \times(r / 2,3 r)$, which is the same number of componenets as $X_{r}$ itself. [Note 25] So if $x$ and $y$ are in the same connected component of $W, \pi(x)$ and $\pi(y)$ are in the same connected component of $X_{r}$.

On the other hand, as mentioned in [ChCo], if $x$ and $y$ are in the same connected component of $X_{r}$, then the points $x_{s}, y_{s} \in b^{-1}(s-\psi r, s+\psi r)$ mentioned in Remark 47, are in the same connected component of $b^{-1}(s-$ $4 \psi r, s+4 \psi r)$. This follows by dividing up any curve, $C$, between $x$ and $y$ in $X_{r}$ into points, $x^{i}$ of distance less than $\psi r$ apart. Then there exist points $x_{s}^{i} \in b^{-1}(s-\psi r, s+\psi r)$ as in Remark 47, such that

$$
\begin{aligned}
d_{W}\left(x_{s}^{i}, x_{s}^{i+1}\right) & <d_{X_{r} \times[r / 2,3 r]}\left(F_{G H}\left(x_{s}^{i}\right), F_{G H}\left(x_{s}^{i+1}\right)\right)+\psi r \\
& <d_{X_{r}}\left(\pi\left(x_{s}^{i}\right), \pi\left(x_{s}^{i+1}\right)\right)+\left|b\left(x_{s}^{i}\right)-b\left(x_{s}^{i+1}\right)\right|+\psi r \\
& <d_{X_{r}}\left(\pi\left(x_{s}^{i}\right), x^{i}\right)+d_{X_{r}}\left(x^{i}, x^{i+1}\right)+d_{X_{r}}\left(x^{i+1}, \pi\left(x_{s}^{i+1}\right)\right)+3 \psi r \\
& <3 \psi r+3 \psi r=6 \psi r
\end{aligned}
$$

Thus a piecewise geodesic from $x_{s}$ to $y_{s}$ can be drawn through these points and will remain in the set $b^{-1}(s-4 \psi r, s+4 \psi r)$.

Proof of Lemma 46. Fix any $\psi \in(0,1 / 2)$. Let $\omega>0$. We will choose the value of $\omega$ later. Let $R_{\psi, \omega}$ be large enough that

$$
\operatorname{Vol}_{n-1}\left(b^{-1}\left(R_{\psi, \omega}(1 / 2-\psi)\right)\right)>(1-\omega) V_{\infty}
$$

where $V_{\infty}=\lim _{R \rightarrow \infty} V o l_{n-1}\left(b^{-1}(R)\right)$ as in (2.3). Thus, by Lemma 36, we have for any $r \geq R_{\psi, \omega}$,

$$
U=b^{-1}(r / 2-\psi r, 3 r+\psi r)
$$

has $\omega$-almost maximal volume [Defn 26] with the distance function

$$
\rho(x)=d\left(x, b^{-1}(3 r+\psi r)\right)
$$

and $f(\rho)=1$. By Lemma 35, $\rho(x)=3 r+\psi r-b(x)$. If we rescale this region by dividing the metric by $r^{2}$, it still has $\omega$-almost maximal volume by Lemma 37 .

The rescaled region, $U$, can be described as the region between two level sets of a distance function, $\bar{\rho}(x)$ which is the rescaling of $\rho(x)$, as follows:

$$
U=\bar{\rho}^{-1}(0,5 / 2+2 \psi) .
$$


By Lemma 39, we have

$$
\begin{aligned}
\operatorname{diam}_{\text {rescaled }}(U) & =\frac{\operatorname{diam}\left(b^{-1}(r / 2-\psi r, 3 r+\psi r)\right.}{r} \\
& \leq \frac{2(5 / 2+2 \psi) r+\operatorname{diam}\left(b^{-1}(3 r+\psi r)\right)}{r} \leq 5+4 \psi+C_{D}
\end{aligned}
$$

where $C_{D}$ is the diameter growth bound in Note 22. Now set the parameters $a=0, b=(5 / 2+2 \psi), f(\bar{\rho})=1, \Lambda=0, D=\left(5+4 \psi+C_{D}\right), \alpha=\psi, \alpha^{\prime}=\psi / 2$, and $\chi=\psi / 4$ and apply the Cheeger-Colding Theorem 27 .

Thus there exists a function,

$$
\Psi(\omega)=\Psi\left(\omega \mid n, \mathbf{1}, a, b, \alpha, \alpha^{\prime}, \chi, \Lambda, D\right),
$$

which converges to 0 as $\omega$ converges to 0 , such that there exists a length space $X_{\psi, U}$, depending on $\alpha^{\prime}=\psi / 2$, and $\chi=\psi / 4$ and $U$, such that

$$
d_{G H}\left(\bar{\rho}^{-1}(\psi, 5 / 2+\psi), X \times(\psi, 5 / 2+\psi)\right)<\Psi(\omega)
$$

where the metric on $r^{-1}(\psi, 5 / 2+\psi)$ is $d_{W}$ where $W=r^{-1}(\psi / 2,5 / 2+3 \psi / 2)$. By Remark 29, the Gromov-Hausdorff map has the form $F_{G H}(x)=$ $(\pi(x), \bar{\rho}(x))$ and by Remark 30, $\pi: \bar{\rho}^{-1}(t) \mapsto X_{\psi, U}$ is also a GromovHausdorff map.

We now choose $\omega_{\psi}$ small enough that $\Psi\left(\omega_{\psi}\right)<\psi$. Rescaling the information back up to full size, we know there exists $R_{\psi}=R_{\psi, \omega_{\psi}}$ of (3.6) such that for all $r \geq R_{\psi}$ there exists $X_{r}$ such that

$$
d_{G H}\left(\rho^{-1}(\psi r,(5 / 2) r+\psi r), X_{r} \times(\psi r,(5 / 2) r+\psi r)\right)<\psi r .
$$

Here the metric is rescaled to $d_{W}$ where $W=\rho^{-1}(\psi / 2 r, 5 r / 2+3 r \psi / 2)$. The Gromov-Hausdorff map has the form $(\pi(x), \rho(x))$ and $\pi: \rho^{-1}(t) \mapsto X_{r}$ is also a Gromov-Hausdorff map. This easily implies the lemma using the fact that $b(x)=3 r+\psi r-\rho(x)$.

Using the above lemma, we can now compare the localized diameters of nearby level sets.

Lemma 49. Let $M^{n}$ have nonnegative Ricci curvature and linear volume growth. Fix $\delta \in(0,1 / 2]$. Given any $\psi \in(0, \delta / 10)$, if $r \geq R_{\psi}$ of Lemma 46 then for any $r_{1}, r_{2} \in[r, 2 r]$ we have,

$$
\mid \operatorname{diam}_{\delta r_{1}}\left(b^{-1}\left(r_{1}\right)\right)-\operatorname{diam}_{\delta r_{2}}\left(b^{-1}\left(r_{2}\right) \mid<6 \psi N_{\delta} r\right.
$$


where $N_{\delta}$ is a uniform upper bound on the number of points $x_{i} \in X_{r}$ such that $d\left(x_{i}, x_{j}\right) \geq(\delta-4 \psi) r$. In particular,

$$
N_{\delta}=\left(\frac{10 C_{D}(1+\delta)}{\delta}\right)^{n}
$$

where $C_{D}$ is the constant bounding diameter growth of Theorem 22 .

Proof. By Lemma 46 we know that

$$
d_{G H}\left(b^{-1}([r / 2,3 r]), X_{r} \times[r / 2,3 r]\right)<\psi r .
$$

We first prove that $N_{\delta}$ is a uniform upper bound on the number of points $x_{i} \in X_{r}$ such that $d\left(x_{i}, x_{j}\right) \geq(\delta-4 \psi) r$.

Let $x_{1}, x_{2} \ldots x_{N}$ be a maximal set of such points in $X_{r}$. By Remark 47 , there exists $y_{i} \in b^{-1}((r-\psi r, r+\psi r))$ such that $d_{X_{r}}\left(\pi\left(y_{i}\right), x_{i}\right)<\psi r$. In particular,

$$
\begin{aligned}
d_{W}\left(y_{i}, y_{j}\right) & >d_{X_{r} \times[r / 2,3 r]}\left(F_{G H}\left(y_{i}\right), F_{G H}\left(y_{j}\right)\right)-\psi r \\
& \geq d_{X_{r}}\left(\pi\left(y_{i}\right), \pi\left(y_{j}\right)\right)-\psi r \\
& \geq d_{X_{r}}\left(x_{i}, x_{j}\right)-d_{X_{r}}\left(\pi\left(y_{i}\right), x_{i}\right)-d_{X_{r}}\left(x_{j}, \pi\left(y_{j}\right)\right)-\psi r \\
& >(\delta-4 \psi) r-3 \psi r=(3 \delta / 10) r .
\end{aligned}
$$

Thus $B_{y_{i}}(\delta r / 10)$ are disjoint balls in $b^{-1}(r-\delta r / 5, r+\delta r / 5)$.

Recall the linear diameter growth constant, $C_{D} \geq 1$, of Theorem 22 . Since $y_{i}$ are in $b^{-1}(r-\delta r / 5, r+\delta r / 5)$, which has diameter less than or equal to $2(\delta r / 5)+C_{D}(r+\delta r / 5)$ by Lemma 39 , we can apply the Relative Volume Comparison Theorem to bound the volumes of the balls from below.

$$
\begin{aligned}
\operatorname{Vol}\left(B_{y_{i}}(\delta r / 10)\right) & \geq \frac{(\delta r / 10)^{n} \operatorname{Vol}\left(B_{y_{i}}\left(C_{D}(r+\delta r / 5)+2 \delta r / 5\right)\right)}{\left(C_{D}(r+\delta r / 5)+2 \delta r / 5\right)^{n}} \\
& \geq\left(\frac{(\delta r / 10)}{C_{D}(r+\delta r)}\right)^{n} \operatorname{Vol}\left(b^{-1}(r-\delta r / 5, r+\delta r / 5)\right) .
\end{aligned}
$$

Since the balls are all disjoint,

$$
\begin{aligned}
\operatorname{Vol}\left(b^{-1}((r-\delta r / 5, r+\delta r / 5))\right) & \geq \sum_{i=1}^{N} \operatorname{Vol}\left(B_{y_{i}}(\delta r / 5)\right) \\
& \geq N \frac{(\delta)^{n} \operatorname{Vol}\left(b^{-1}(r-\delta r / 5, r+\delta r / 5)\right)}{\left(10 C_{D}(1+\delta)\right)^{n}}
\end{aligned}
$$


and we have the uniform estimate for $N_{\delta}$.

We now prove that for all $s \in[r, 2 r]$,

$$
\operatorname{diam}_{\delta s}\left(b^{-1}(s)\right)<\operatorname{diam}\left(X_{r}\right)+3 \psi N_{\delta} r,
$$

where $\operatorname{diam}\left(X_{r}\right)$ is the diameter of the largest connected component of $X_{r}$.

Recall Definition 44 of the almost intrinsic diameter. Let $U=b^{-1}(s-$ $\delta s, s-\delta s)$ and let $V$ be the largest pathwise connected component of $U$; so

$$
\operatorname{diam}_{V}\left(b^{-1}(s) \cap V\right)=\operatorname{diam}_{\delta s}\left(b^{-1}(s)\right) .
$$

Let $x$ and $y$ be any pair of points in $b^{-1}(s) \cap V$. We claim that for all $\varepsilon>0$, there exists a curve, $c$, contained in $V$ from $x$ to $y$ such that

$$
L(c)<\operatorname{diam}\left(X_{r}\right)+3 N_{\delta} \psi r+\varepsilon .
$$

Once we have proven the claim, (3.20) is proven.

Fix $\varepsilon$. To find a curve, $c$, we first note that $x$ and $y$ are in the same connected component of $b^{-1}(r / 2,3 r)$. Thus $\pi(x)$ and $\pi(y)$ are in the same connected componenet of $X_{r}$. So there exists a curve, $C_{\varepsilon}$, in the length space $X_{r}$ between $\pi(x)$ and $\pi(y)$ such that

$$
d_{X_{r}}(\pi(x), \pi(y)) \leq L\left(C_{X}\right) \leq d_{X_{r}}(\pi(x), \pi(y))+\varepsilon .
$$

Let $p_{0}=\pi(x), p_{1}, p_{2}, \ldots p_{N}=\pi(y)$ be equally spaced points along $C_{\varepsilon}$ such that $N=N_{\delta}+1$. Thus $d\left(p_{i}, p_{i+1}\right)<(\delta-4 \psi) r$ and

$$
\sum_{i=0}^{N-1} d_{X_{r}}\left(p_{i}, p_{i+1}\right) \leq L\left(C_{\varepsilon}\right) .
$$

By Remark 47, there exists $x_{i} \in b^{-1}((s-\psi r, s+\psi r))$ such that $x_{0}=x$, $x_{N}=y$ and $d_{X_{r}}\left(\pi\left(x_{i}\right), p_{i}\right)<\psi r$. Thus,

$$
\begin{aligned}
d\left(x_{i}, x_{i+1}\right) & \leq d_{W}\left(x_{i}, x_{i+1}\right)<d_{X_{r}}\left(\pi\left(x_{i}\right), \pi\left(x_{i+1}\right)\right)+\psi r \\
& <3 \psi r+d_{X_{r}}\left(p_{i}, p_{i+1}\right)<\delta r-\psi r<\delta s,
\end{aligned}
$$

where $W=b^{-1}(r / 2-\psi r / 2,3 r+\psi r / 2)$ as in Lemma 46. So a minimal geodesic from $x_{i}$ to $x_{i+1}$ is contained in $b^{-1}((s-\delta s, s+\delta s)) \subset W$. So there is a piecewise minimal geodesic curve from $x$ to $y$ contained in $b^{-1}((s-\delta s, s+\delta s))$ of length,

$$
\begin{aligned}
\sum_{i=0}^{N-1} d_{W}\left(x_{i}, x_{i+1}\right) & \leq \sum_{i=0}^{N-1} d_{X_{r}}\left(p_{i}, p_{i+1}\right)+N_{\delta} 3 \psi r \\
& \leq L\left(C_{\varepsilon}\right)+3 N_{\delta} \psi r \\
& \leq \operatorname{diam}\left(X_{r}\right)+\varepsilon+3 N_{\delta} \psi r .
\end{aligned}
$$


This piecewise geodesic satisfies our claim, (3.22), and (3.20) follows.

We now prove that for all $s \in[r, 2 r]$,

$$
\operatorname{diam}\left(X_{r}\right)<\operatorname{diam}_{\delta s}\left(b^{-1}(s)\right)+3 \psi r .
$$

First recall that $\operatorname{diam}\left(X_{r}\right)$ is the diameter of its largest connected component, $Z_{r} \subset X_{r}$. Let $x$ and $y$ be any pair of points in $Z_{r}$. There exists $p, q \in b^{-1}((s-\psi r, s+\psi r))$ such that

$$
d(\pi(p), x)<\psi r \text { and } d(\pi(q), y)<\psi r,
$$

as mentioned in Remark 47.

Now, $p$ and $q$ are in the same connected component of $b^{-1}((s-\delta s, s+\delta s))$ by Remark 48 and by the fact that $b^{-1}((s-\delta s, s+\delta s))$ contains the region $b^{-1}((s-4 \psi r, s+4 \psi r))$. So for all $\varepsilon>0$, there exists a curve $C_{\varepsilon} \in b^{-1}((s-$ $\delta s, s+\delta s)) \subset W$ which almost achieves the $s \delta$-almost intrinsic distance between $p$ and $q$ and

$$
d_{W}(p, q) \leq L\left(C_{\varepsilon}\right) \leq d_{s \delta}(p, q)+\varepsilon \leq \operatorname{diam}_{\delta s}\left(b^{-1}(s)\right)+\varepsilon .
$$

On the other hand, by (3.15),(3.26) and the triangle inequality,

$$
\begin{aligned}
d_{W}(p, q) & \geq d_{X_{r} \times[r / 2,3 r]}\left(F_{G H}(p), F_{G H}(q)\right)-\psi r \\
& \geq d_{X_{r}}(\pi(p), \pi(q))-\psi r \geq d_{X_{r}}(x, y)-3 \psi r .
\end{aligned}
$$

Combining (3.27) and (3.28), taking $\varepsilon \rightarrow 0$ and then maximizing over all $x$ and $y$ in $Z_{r}$, we have (3.25).

It is easy to see that (3.20) and (3.25) applied to $s=r_{1}$ and $s=r_{2}$ alternatively, imply the lemma.

We can now prove the sublinear almost intrinsic diameter growth theorem.

Proof of Theorem 45. We need to show that given any $\delta \in(0,1 / 2)$, we have sublinear almost intrinsic diameter growth,

$$
\lim _{R \rightarrow \infty} \frac{\operatorname{diam}_{\delta R}\left(b^{-1}(R)\right)}{R}=0 .
$$

Given any $\varepsilon>0$, let

$$
\psi=\min \left\{\delta / 10, \varepsilon / 3 N_{\delta}\right\}
$$


Thus by Lemmas 46 and 49, there exists $R_{\psi}$ such that for all $r \geq R_{\psi}$ and for all $r^{\prime} \in[r, 2 r]$,

$$
\operatorname{diam}_{\delta r^{\prime}}\left(b^{-1}\left(r^{\prime}\right)\right)<\varepsilon r+\operatorname{diam}_{\delta r}\left(b^{-1}(r)\right) .
$$

Thus, applying this repeatedly to $r=2^{j} \delta R_{\varepsilon}$ and $r^{\prime}=2 r$, we have

$$
\begin{aligned}
\operatorname{diam}_{2^{k} \delta R_{\varepsilon}}\left(b^{-1}\left(2^{k} R_{\varepsilon}\right)\right. & <\varepsilon 2^{k-1} R_{\varepsilon}+\operatorname{diam}_{\delta 2^{k-1} R_{\varepsilon}}\left(b^{-1}\left(2^{k-1} R_{\varepsilon}\right)\right) \\
& <\varepsilon 2^{k-1} R_{\varepsilon}+\varepsilon 2^{k-2} R_{\varepsilon}+\operatorname{diam}_{\delta 2^{k-2} R_{\varepsilon}}\left(b^{-1}\left(2^{k-2} R_{\varepsilon}\right)\right) \\
& <\varepsilon\left(2^{k-1}+\ldots+2+1\right) R_{\varepsilon}+\operatorname{diam}_{\delta R_{\varepsilon}}\left(b^{-1}\left(R_{\varepsilon}\right)\right) \\
& <\varepsilon 2^{k} R_{\varepsilon}+\operatorname{diam}_{\delta R_{\varepsilon}}\left(b^{-1}\left(R_{\varepsilon}\right)\right)
\end{aligned}
$$

For all $R>R_{\varepsilon}$ there exists $k$ such that $R \in\left[2^{k} R_{\varepsilon}, 2^{k+1} R_{\varepsilon}\right]$ so by (3.31), we have

$$
\frac{\operatorname{diam}_{\delta R}\left(b^{-1}(R)\right)}{R}<\varepsilon+2 \frac{\operatorname{diam}_{2^{k} \delta R_{\varepsilon}}\left(b^{-1}\left(2^{k} R_{\varepsilon}\right)\right)}{2^{k} R_{\varepsilon}}
$$

Combining this with the above estimate and taking $R$ to infinity, we get

$$
\limsup _{R \rightarrow \infty} \frac{\operatorname{diam}_{\delta R}\left(b^{-1}(R)\right)}{R} \leq \varepsilon+2 \lim _{k \rightarrow \infty} \frac{\varepsilon 2^{k} R_{\varepsilon}+\operatorname{diam}_{\delta R_{\varepsilon}}\left(b^{-1}\left(R_{\varepsilon}\right)\right)}{2^{k} R_{\varepsilon}}=3 \varepsilon .
$$

Since this is true for all $\varepsilon>0$ we have sublinear diameter growth.

We now prove our final theorem, Theorem 1 , that

$$
\lim _{R \rightarrow \infty} \frac{\operatorname{diam}\left(b^{-1}(R)\right)}{R}=0 .
$$

Proof of Theorem 1. By Theorem 22 of [So2], we already know that

$$
\frac{\operatorname{diam}\left(b^{-1}(R)\right)}{R} \leq C_{D}<\infty .
$$

If we assume that the diameter growth is not sublinear, then there exists a sequence, $r_{i}$, approaching infinity such that

$$
\frac{\operatorname{diam}\left(b^{-1}\left(r_{i}\right)\right)}{r_{i}} \geq C_{L}>0 \quad \forall i .
$$

So there exist $x_{i}, y_{i} \in b^{-1}\left(r_{i}\right)$, and there exists, $\sigma_{i}$, a minimal geodesic from $x_{i}$ to $y_{i}$ such that $L\left(\sigma_{i}\right)=h_{i} r_{i}$ where $h_{i} \in\left[C_{L}, C_{D}\right]$. 
Suppose there is a subsequence, $i_{j}$, such that

$$
\sigma_{i_{j}} \subset b^{-1}\left(\left[r_{i_{j}} / 2,3 r_{i_{j}} / 2\right]\right) .
$$

Then we have a minimal geodesic of length $h_{i_{j}} r_{i_{j}}$ contained in this region, so

$$
\operatorname{diam}_{\frac{1}{2}\left(r_{i_{j}}\right)}\left(b^{-1}\left(r_{i_{j}}\right)\right) \geq h_{i_{j}} r_{i_{j}} \geq C_{L} r_{i_{j}}
$$

This contradicts the sublinear $1 / 2$-almost intrinsic diameter growth of the manifold [Theorem 45]. Thus there exists $N$ such that for all $i \geq N, \sigma_{i}$ is not a subset of $b^{-1}\left(r_{i} / 2,3 r_{i} / 2\right)$.

Let

$$
S_{i}=\min _{t \in\left[0, h_{i} r_{i}\right]} b\left(\sigma_{i}(t)\right)
$$

and let

$$
T_{i}=\max _{t \in\left[0, h_{i} r_{i}\right]} b\left(\sigma_{i}(t)\right) .
$$

For all $i \geq N$ either $2 S_{i}<r_{i}$ or $2 T_{i} / 3>r_{i}$.

Suppose there is a subsequence, $i_{j}$, such that $2 T_{i_{j}} / 3>r_{i_{j}}$. Then $\sigma_{i_{j}}$ is a minimal geodesic which starts at $x_{i_{j}}$ in $b^{-1}\left(r_{i_{j}}\right)$, passes through a point, $x_{i_{j}}^{\prime}$ in $b^{-1}\left(2 T_{i_{j}} / 3\right)$, passes through a point in $b^{-1}\left(T_{i_{j}}\right)$, continues back through a point, $y_{i_{j}}^{\prime}$ in $b^{-1}\left(2 T_{i_{j}} / 3\right)$, before returning to $y_{i_{j}}$ in $b^{-1}\left(r_{i_{j}}\right)$. Thus there are points $x_{i_{j}}^{\prime}$ and $y_{i_{j}}^{\prime}$ in $b^{-1}\left(2 T_{i_{j}} / 3\right)$ with a minimal geodesic between them of length at least $2 T_{i_{j}} / 3$ which remains in $b^{-1}\left(2 T_{i_{j}} / 3, T_{i_{j}}\right)$. So

$$
\operatorname{diam}_{\frac{1}{2}\left(2 T_{i_{j}} / 3\right)}\left(b^{-1}\left(2 T_{i_{j}} / 3\right)\right) \geq 2 T_{i_{j}} / 3 .
$$

As $r_{i_{j}}$ goes to infinity, $T_{i_{j}}$ approaches infinity, and then (3.40) contradicts Theorem 45 with $\delta=1 / 2$. Thus there exists $N^{\prime}$ such that $2 T_{i} / 3 \leq r_{i}$ for all $i \geq N^{\prime} ;$ so $2 S_{i}<r_{i}$ for all $i \geq N^{\prime}$.

We would like to use the same trick with the $S_{i}$ of (3.38). but first we must show that $S_{i}$ diverge to infinity.

Suppose there exists $R$, and there exists a subsequence $i_{j}$ such that $S_{i_{j}} \leq R$. Thus there exists $t_{i_{j}}$ such that $\sigma_{i_{j}}\left(t_{i_{j}}\right) \in b^{-1}(R)$. Since this level set is compact, a subsequence of the $\sigma_{i_{j}}$ must converge. Since $L\left(\sigma_{i_{j}}\right) \geq C_{L} r_{i_{j}}$, this subsequence must converge to a line. So by the Splitting Theorem of Cheeger and Gromoll, the manifold is split [ChGl]. However, this implies that $b^{-1}\left(r_{i}\right)$ is totally geodesic and so $S_{i}=r_{i}$, which contradicts $2 S_{i}<r_{i}$. 
Thus $S_{i}$ diverges to infinity and $S_{i} \leq r_{i} / 2$. Then $\sigma_{i}$ is a minimal geodesic which starts at $x_{i}$ in $b^{-1}\left(r_{i}\right)$, passes through a point, $x_{i}^{\prime}$ in $b^{-1}\left(2 S_{i}\right)$, passes through a point in $b^{-1}\left(S_{i}\right)$, continues back through a point, $y_{i}^{\prime}$ in $b^{-1}\left(2 S_{i}\right)$, before returning to $y_{i}$ in $b^{-1}\left(r_{i}\right)$. Thus there are points $x_{i}^{\prime}$ and $y_{i}^{\prime}$ in $b^{-1}\left(2 S_{i}\right)$ with a minimal geodesic between them of length at least $2 S_{i}$ which remains in $b^{-1}\left(S_{i}, 2 S_{i}\right)$. So

$$
\operatorname{diam}_{\frac{1}{2}\left(2 S_{i}\right)}\left(b^{-1}\left(2 S_{i}\right)\right) \geq 2 S_{i},
$$

which contradicts Theorem 22 for $\delta=1 / 2$ as $S_{i}$ approaches infinity.

Thus $C_{L}$ of (3.35) cannot exist, and the manifold has sublinear diameter growth.

Similar theorems may be provable for manifolds with a quadratically decaying lower bound on Ricci curvature, but one must be careful to rescale $X_{r}$ as it is compared to each level set.

\section{References.}

[AbGl] U. Abresch and D. Gromoll, On complete manifolds with nonnegative Ricci curvature, J. Amer. Math. Soc. 3 (1990), no. 2, 355-374.

[Bi] R. Bishop, A Relation Between Volume, Mean Curvature and Diameter, Amer. Math. Soc. Not. 10 (1963), 364.

[BiCr] R. Bishop and R. Crittenden, Geometry of Manifolds, Pure and Applied Mathematics, Vol. XV. Academic Press, New York-London 1964.

[Boc] S. Bochner, Vector fields and Ricci curvature, Bull. Amer. Math. Soc. 52, (1946). 776-797.

[Bu] H. Busemann, The Geometry of Geodesics, Academic Press Inc., New York, N. Y., 1955.

[doC] M.P. do Carmo, Riemannian Geometry, Translated by Francis Flaherty. Mathematics: Theory \& Applications. Birkhauser Boston, Inc., Boston, MA, 1992.

[Ch] J. Cheeger, Critical points of distance functions and applications to geometry, Geometric topology: recent developments (Montecatini Terme, 1990), 1-38, Lecture Notes in Math., 1504, Springer, Berlin, 1991.

[ChCo] J. Cheeger and T. Colding, Lower bounds on Ricci curvature and the almost rigidity of warped products, Annals of Math. (2) 144 (1996), no. 1, 189-237. 
[ChEb] J. Cheeger and D. Ebin, Comparison Theorems in Riemannian Geometry, North-Holland Mathematical Library, Vol. 9. North-Holland Publishing Co., Amsterdam-Oxford; American Elsevier Publishing Co., Inc., New York, 1975. viii+174 pp.

[ChGl] J. Cheeger and D. Gromoll, The splitting theorem for manifolds of nonnegative Ricci curvature, J. Differential Geometry, 6 (1971/72), 119128.

[ChGrTa] J. Cheeger, M. Gromov and M. Taylor, Finite propagation speed, kernel estimates for functions of the Laplace operator, and the geometry of complete Riemannian manifolds, J. Differential Geom. 17 (1982), no. $1,15-53$.

[CgYau] S.Y. Cheng and S.T. Yau, Differential Equations on Riemannian manifolds and their geometric applications, Comm. Pure Appl. Math. 28 (1975) 333-354.

[Co1] T. Colding, Stability and Ricci curvature, C. R. Acad. Sci. Paris Sr. I Math. 320 (1995), no. 11, 1343-1347.

[Co2] T. Colding, Large Manifolds with Positive Ricci Curvature, Invent. Math. 124 (1996), 193-214.

[Fed] H. Federer, Geometric Measure Theory, Die Grundlehren der mathematischen Wissenschaften, Band 153 Springer-Verlag New York Inc., New York 1969.

[GrLaPa] M. Gromov, J. Lafontaine and P. Pansu, Structures Métriques pour Variétés Riemanniennes, (French) Textes Mathématiques, 1. CEDIC, Paris, 1981.

[So1] C. Sormani, Complete Noncompact Manifolds with Lower Bounds on Ricci Curvature and Minimal Volume Growth, PhD Thesis, Courant Institute (1996).

[So2] C. Sormani, Busemann Functions on Manifolds with Lower Bounds on Ricci Curvature and Minimal Volume Growth, Journal of Differential Geometry 48, (1998) 557-585.

[So3] C. Sormani, Harmonic Functions on Manifolds with Nonnegative Ricci Curvature and Linear Volume Growth, preprint. To appear in the Pacific Journal of Mathematics.

[Yau] S. T. Yau, Harmonic functions on complete Riemannian manifolds, Comm. Pure Appl. Math. 28 (1975), 201-228. 
RECEIVED JANUARY 6, 1998.

JOHNS HOPKINS UNIVERSITY

3400 North Charles StreEt

BALTIMORE MD 21218

E-mail address: sormanic@member.ams.org 